\title{
因HAD
}

ISSN-L: 2530-5115

(c) (1)(2)

DOI: http://doi.org/10.22585/hospdomic.v4i4.113

\section{Intervenciones desde la farmacia comunitaria en los pacientes adultos que reciben atención de la salud a domicilio: revisión exploratoria}

Community pharmacy interventions in adult patients receiving home health care: an exploratory review

Jessica Sánchez-Moya', Javier Sanz-Valero ${ }^{2,3}$, Elsa López-Pintor ${ }^{4}$

1. Universidad Miguel Hernández. Facultad de Farmacia, Campus de Sant Joan d'Alacant. Alicante. España.

2. Instituto de Salud Carlos III. Escuela Nacional de Medicina del Trabajo. Madrid. España.

3. Universidad Miguel Hernández. Departamento de Salud Pública e Historia de la Ciencia, Campus de Sant Joan d'Alacant. Alicante. España.

4. Universidad Miguel Hernández. Departamento de Ingeniería, Área de Farmacia y Tecnología Farmacéutica. Campus de Sant Joan d'Alacant. Alicante. España.

Correspondencia/Correspondence

Elsa López-Pintor

elsa.lopez@umh.es

Recibido/Received

20.07.2020

Aceptado/Accepted

18.08.2020
Conflicto de Intereses/Competing interest Las autoras y el autor del trabajo declaran la inexistencia de conflicto de interés.

CÓMO CITAR ESTE TRABAJO | HOW TO CITE THIS PAPER

Sánchez-Moya J, Sanz-Valero J, López-Pintor E. Intervenciones desde la farmacia comunitaria en los pacientes adultos que reciben atención de la salud a domicilio: revisión exploratoria. Hosp Domic. 2020;4(4):209-27. 


\section{RESUMEN}

Objetivos: Revisar la literatura científica relacionada con las intervenciones desde la farmacia comunitaria en los pacientes adultos que recibieron atención de la salud a domicilio.

Métodos: Revisión exploratoria de los artículos recuperados de las bases de datos bibliográficas MEDLINE (PubMed), Embase, Cochrane Library, Scopus y Web of Science hasta marzo de 2020. La ecuación de búsqueda se formuló mediante los descriptores "Home Care Services" y "Pharmacies" o "Community Pharmacy Services", utilizando también los Entry Terms relacionados y los filtros: «Humans» y «Adult: 19+ years». La calidad de los artículos se evaluó mediante el cuestionario STROBE.

Resultados: De las 307 referencias recuperadas, tras aplicar los criterios de inclusión y exclusión, se seleccionaron 44 artículos: 11 estudios descriptivos transversales y 10 ensayos aleatorizados controlados. En 19 (43\%) de estos se observó que las intervenciones realizadas desde la farmacia comunitaria aumentaron la adherencia fármaco-terapéutica. También, un mejor conocimiento sobre los medicamentos (administración, seguridad, dosis, posología) en 17 (38,6\%) trabajos y en 13 (29,5\%) estudios se consiguió la prevención o resolución de los PRM. Al evaluar la calidad de los artículos seleccionados para la revisión mediante el cuestionario STROBE, las puntuaciones oscilaron entre un mínimo de 11 y un máximo de 20,8 sobre una puntuación máxima de 22

Conclusiones: La intervención farmacéutica, a través de la farmacia comunitaria, aportó beneficios en la población más adulta, mediante el aumento de la adherencia farmacoterapéutica, la disminución de los problemas relacionados con los medicamentos y la mejora en el almacenamiento y conservación de los mismos.

Palabras clave: Servicios de Atención de Salud a Domicilio; Farmacias; Servicios Comunitarios de Farmacia; Cumplimiento y Adherencia al Tratamiento; Errores de Medicación; Educación del Paciente como Asunto; Polifarmacia; Ancianos; Satisfacción del Paciente.

\section{ABSTRACT}

Objective: To review the scientific literature related to interventions from the community pharmacy in adult patients who received home health care.

Method: Exploratory review of the articles retrieved from the bibliographic databases MEDLINE (PubMed), Embase, Cochrane Library, Scopus and Web of Science until March 2020. The search equation was formulated using the descriptors "Home Care Services" and "Pharmacies" or "Community Pharmacy Services", also using the related Entry Terms and filters: "Humans" and "Adult: 19+ years". The quality of the articles was evaluated using the STROBE questionnaire.

Results: From the 307 references retrieved, after applying the inclusion and exclusion criteria, 44 articles were selected: 11 cross-sectional descriptive studies and 10 randomized controlled trials. In 19 (43\%) of these, it was observed that the interventions carried out by the community pharmacy increased drug-therapeutic adherence. Also, a better knowledge of medications (administration, safety, dosage, dosage) in 17 $(38.6 \%)$ studies and in $13(29.5 \%)$ studies, the prevention or resolution of DRM was achieved. Once the articles were evaluated by means of the STROBE questionnaire for this review the scores ranged from a minimum of 11 to a maximum of 20.8 out of a maximum score of 22 .

Conclusions: Pharmaceutical intervention, through community pharmacy, brought benefits in the older adult population, by increasing pharmacotherapeutic adherence, reducing drugrelated problems, and challenges in preserving and storing medications.

Keywords: Home Care Services; Pharmacies; Community Pharmacy Services; Treatment Adherence and Compliance; Medication Errors; Patient Education as Topic; Polypharmacy; Aged; Patient Satisfaction. 


\section{INTRODUCCIÓN}

La hospitalización a domicilio ( $\mathrm{HaD}$ ) se definió como el servicio proporcionado, por profesionales sanitarios, que provee de tratamiento activo de la salud en el hogar del paciente, por una afección que de otro modo requeriría ingreso hospitalario (1). Por otra parte, el desarrollo de los servicios hospitalarios en el hogar tiene también como objetivo mejorar la percepción del paciente respecto al tratamiento y mejorar su calidad de vida al ofrecer la posibilidad de dicha atención en un ambiente amigable y con la misma eficiencia en la atención (2). Así, la HaD se ha desarrollado y adaptado a este nuevo contexto siendo en estos momentos una alternativa real para el tratamiento en el domicilio de muchos pacientes con procesos médicos o quirúrgicos que no precisan de toda la estructura de una unidad de hospitalización (3).

En este contexto, la intervención del farmacéutico comunitario en el seguimiento de la medicación de los pacientes, e incluso su participación en los posibles programas de formación, tanto dirigidos a los enfermos como a sus cuidadores, pueden ser clave para mejorar el patrón de la HaD (4). Por ejemplo, la revisión y seguimiento de la medicación de los enfermos ingresados en HaD ofreció una plataforma de colaboración, entre los diferentes sanitarios, que influyó en la mejora y eficacia de los medicamentos utilizados. Costa et al. (5) demostraron que las acciones desde la farmacia comunitaria ayudaron a mejorar la seguridad del paciente, minimizaron los problemas relacionados con la medicación y animaron a los pacientes a desempeñar un papel activo en la gestión de su propia salud.

Kucukarslan et al. (6), demostraron que en aquellos pacientes ingresados en $\mathrm{HaD}$, con bajo nivel de alfabetización en salud que fueron diagnosticados con una enfermedad crónica, se beneficiaron de un seguimiento personalizado e intensivo. Los farmacéuticos ayudaron en la toma de decisiones para la farmacoterapia óptima y realizaron un adecuado seguimiento de la medicación. Este estudio, observó que el farmacéutico comunitario apoyó la transferencia de la atención a la salud desde el hospital a la comunidad. Y, se comprobó el impacto de las intervenciones relacionadas con el seguimiento y adherencia a la medicación en la atención al paciente, mediante resultados medibles, principalmente en aquellos recién diagnosticados con una enfermedad crónica o que aún no habían logrado su objetivo terapéutico.

En la misma línea, Haynes et al. (7), demostraron la importancia de la participación del farmacéutico comunitario ya que, en los países desarrollados, la adherencia a la medicación de los enfermos crónicos en el domicilio era muy deficiente.

La atención farmacéutica domiciliaria es la respuesta profesional y responsable a un futuro marcado por el envejecimiento de la población, la cronicidad, la dependencia y la necesidad de dar respuesta a la continuidad asistencial a estos pacientes.

En consecuencia, el objetivo de este trabajo fue revisar la literatura científica relacionada con las intervenciones desde la farmacia comunitaria en los pacientes adultos que recibieron atención de la salud a domicilio.

\section{MÉTODOS}

\section{Diseño}

Estudio descriptivo transversal y análisis crítico de los trabajos recuperados mediante revisión sistemática. 


\section{Fuente de obtención de los datos}

Los datos se obtuvieron de la consulta directa y acceso, vía Internet, a las siguientes bases de datos bibliográficas del ámbito de las ciencias de la salud: MEDLINE (vía PubMed), Embase, Cochrane Library, Scopus y Web of Science.

\section{Unidad de análisis}

Se trabajó con los artículos publicados y recuperados desde la base de datos bibliográfica indicada.

Para evitar posibles duplicidades en caso de publicación posterior como original u otro tipo documental, no se tuvieron en cuenta las comunicaciones a congresos, aunque sean de gran importancia para la comunidad investigadora ya que contribuyen a la rápida comunicación y difusión de la investigación.

\section{Tratamiento de la información}

Para definir los términos de la búsqueda se consultó el Thesaurus desarrollado por la U.S. National Library of Medicine. Se consideró adecuado el uso de los términos «Home Care Services» y «Pharmacies» O "Community Pharmacy Services», como descriptores y como texto en los campos de registro del título y el resumen, conformando la sintaxis de búsqueda final mediante la intersección booleana de dos ecuaciones: (Ecuación 1) AND (Ecuación 2).

- Ecuación 1 - Servicios de cuidado domiciliario:

"Home Care Services"[MeSH] OR "Home Care"[Title/Abstract] OR "Domiciliary Care"[Title/ Abstract] OR "Home Care Services, Hospital- Based"[Mesh] OR "Hospital-Based Home Care"[All Fields] OR "Hospital Based Home Care"[Title/Abstract] OR "Hospital Home Care Services"[Title/ Abstract] OR "Hospital-Based Home Care Services"[Title/Abstract] OR "Hospital Based Home Care Services"[Title/Abstract] OR "Home Hospitalization"[Title/Abstract] OR "Hospital at Home"[Title/Abstract] OR "Hospital-at-Home"[Title/Abstract] OR "Hospital Home Care"[Title/Abstract] OR "Hospital at Home Care"[Title/Abstract] OR "Hospital in the Home"[Title/Abstract]

- Ecuación 2 Farmacia comunitaria:

"Pharmacies"[Mesh] OR "Community Pharmacy"[Title/Abstract] OR "Community Pharmacies"[Title/Abstract] "Community Pharmacist"[Title/Abstract] OR "Community Pharmacy Services"[Mesh] OR "Community Pharmacy Service"[Title/Abstract] OR "Community Pharmaceutic Service"[Title/Abstract] OR "Community Pharmaceutical Service"[Title/Abstract]

La ecuación de búsqueda final se desarrolló para su empleo en la base de datos MEDLINE, vía PubMed, utilizando los filtros: «Humans» y «Adult: 19+ years».

Esta estrategia se adaptó a las características de cada una del resto de bases de datos consultadas. La búsqueda se realizó desde la primera fecha disponible, de acuerdo a las características de cada base de datos, hasta marzo de 2020 y se completó con el examen del listado bibliográfico de los artículos que fueron seleccionados.

\section{Selección final de los artículos}

Se escogieron para la revisión y análisis crítico los artículos que cumplieron los siguientes criterios: adecuarse a los objetivos de la búsqueda (intervención desde la farmacia comunitaria), estar publicados en revistas arbitradas por pares y escritos en inglés, español o portugués. 
Se excluyeron: aquellos artículos que, además, no se pudo encontrar el texto completo del mismo, no existió relación entre la intervención y el resultado a estudio y los que incluían población no adulta.

La selección de los artículos pertinentes se realizó de forma independiente por los autores: JSM y JSV. Para dar por válida la inclusión de los estudios se estableció que la valoración de la concordancia entre estos autores (índice Kappa) debía ser superior al 60\% (fuerza de la concordancia buena). Siempre que se cumpliera esta condición, las posibles discordancias se solucionaron, previa consulta con la tercera autora ELP, mediante el consenso entre los autores.

La calidad de los artículos seleccionados se valoró utilizando como apoyo las pautas para informar los estudios observacionales STROBE (STrengthening the Reporting of Observational studies in Epidemiology) (8), que contiene una lista de 22 puntos de control esenciales que deben describirse durante la publicación de estos documentos. Para cada artículo seleccionado, se asignó un punto por cada ítem presente (en caso de no ser aplicable, no puntuaba). Cuando un ítem estaba compuesto por varios puntos, estos se evaluaron de forma independiente, dándole el mismo valor a cada uno de ellos y posteriormente se realizó un promedio (siendo éste el resultado final de ese ítem), de tal forma que en ningún caso se pudiera superar la valoración total de un punto por ítem.

Para conocer el nivel de evidencia y su grado de recomendación se utilizaron las recomendaciones de la U. S. Agency for Healthcare Research and Quality (AHRQ) (9).

\section{Extracción de los datos}

El control de la corrección de los datos se realizó mediante dobles tablas que permitieron la detección de las desviaciones y su subsanación mediante nueva consulta de los originales.

Para determinar la actualidad de los artículos se calculó el semiperíodo de Burton-Kebler (la mediana de la edad) y el Índice de Price (porcentaje de artículo con edad inferior a los 5 años).

Los estudios se agruparon según las variables a estudio, con el fin de sistematizar y facilitar la comprensión de los resultados, considerando los siguientes datos: primer autor y año de publicación, diseño del estudio, población incluida en los artículos, país, periodo de implementación de la intervención y resultado principal obtenido en el estudio.

\section{RESULTADOS}

Al aplicar los criterios de búsqueda se recuperaron un total de 330 artículos: $30(9,1 \%)$ en MEDLINE (vía PubMed), 61 (18,5\%) en Embase, 3 (0,9\%) en Cochrane Library, 125 (37,9\%) en Scopus, 90 $(27,3 \%)$ en Web of Science y 23 (7\%) mediante búsqueda manual en los listados bibliográficos de los artículos seleccionados.

Tras depurar los 96 (29,1\%) registros repetidos, aplicar los criterios de inclusión y exclusión, fue posible seleccionar 44 (13,3\%) artículos (10-53), para su revisión y análisis crítico; ver figura 1 y tabla 1.

A la hora de revisar los artículos se observó que existían estudios que, si bien presentaron diferentes objetivos de estudio o poblaciones examinadas, los resultados relacionados con la intervención farmacéutica no diferían entre ellos y, por lo tanto, solo se aceptó el más actual para su revisión. Este fue el caso de Toivo et al. $(13,54)$, Stafford et al. $(33,55)$, Papastergiou et al. $(11,56)$, Holland et al. (39,57-59), Roughead et al. $(35,60)$ y Stewart et al. $(51,61)$. 
El acuerdo sobre la pertinencia de los estudios seleccionados, entre los dos evaluadores, calculado mediante el índice Kappa fue del 100\%.

Los artículos elegidos presentaron una obsolescencia, según el Índice de Burton Kebler, igual a 8 años, con un Índice de Price del 27,3\%. El año con mayor número de trabajos publicados fue el 2012, con 8 publicaciones (27-34).

Al evaluar la calidad de los artículos seleccionados para la revisión, mediante el cuestionario STROBE, las puntuaciones oscilaron entre mínimo de 11,0 (55\% cumplimiento) y máximo de 20,8 (94,5\% cumplimiento) con mediana igual a 14,3 (tabla 2).

Según los criterios de la U. S. Agency for Healthcare Research and Quality (AHRQ) esta revisión presentó evidencia lb (procede de al menos un ensayo clínico aleatorizado) con grado de recomendación B (basada en estudios de las categorías lb, Ila y IIb).

El diseño predominante en los 44 trabajos revisados fue en 11 de ellos descriptivo transversal $(11,14,23,24,26,27,29,38,42,46,50)$ y en 10 , de ensayo aleatorizado controlado $(13,20,39,41,43-$ $45,47,48,61)$.

La filiación mayoritaria de los trabajos incluidos en la revisión fue estadounidense, con 16 artículos (11,15-17,21,22,24-26,36,38,41,43,46,51,52), australiana con 10 artículos $(18,23,30,31,33,35,42,44,45,47)$ y británica con 7 (14,39,40,48-50,53); el estudio de Fikri-Benbrahim et al. (32), presentó filiación española. Todos los trabajos aceptados en esta revisión estuvieron redactados en inglés.

La población incluida en los trabajos recopilados en esta revisión osciló entre un mínimo de 10 personas (22) y un máximo de 17136 (35), situándose el rango de la edad entre 49 y 88 años, excepto los estudios de Reidt et al. (25) y El Hajj et al. (28) que incluyeron una población entre los 18 y 65 años de edad. Se comprobó que el trabajo de Clifton et al. (16) no se informaba sobre la separación, según sexo, de la población. Y, en los artículos de Fujita et al. (12), Elliott et al. (18), Triller et al. (46) y Nazaret et al. (48), no constaba ni la edad ni el sexo de la población a estudio.

En el estudio de Reidt et al. (25) se comprobó que en el grupo control se habían seleccionado 227 pacientes más que en el grupo intervención. Si bien, lo más destacado se pudo probar en el artículo de Roughead et al. (35) donde en el grupo de intervención se constató la existencia de 816 pacientes, mientras que en el grupo control habían 16.320.

Los pacientes incluidos fueron principalmente ancianos, crónicos y/o polimedicados (10-16,1821,25-27,29-32,34,36-38,42,44,46,47,49,51-53). 4 estudios incluyeron pacientes al alta hospitalaria $(14,24,40,48)$ y 10 artículos se centraron en pacientes con patologías concretas, como los desórdenes cardiovasculares (17), insuficiencia cardiaca $(22,39,41)$, hipertensión arterial (43), dislipemias (45) o dolor crónico. Dos estudios se realizaron en pacientes anticoagulados $(33,35)$ y en dos artículos no se especificaba alguna patología o circunstancia concreta en los pacientes $(23,28)$.

El periodo temporal que presentaron los estudios osciló entre los 2 y los 31 meses $(27,35)$, variando el periodo de reclutamiento, también, entre los 2 meses y los 31 meses $(12,29)$. El periodo de seguimiento fluctuó entre 1 mes $(10,19,53)$ y 15 meses (18) y el de recopilación de los datos varió de 5 a 7 meses $(15,23)$. Los artículos de Sorensen et al. (42) y de Raynor et al. (49) no indicaron ningún periodo.

\section{Intervenciones realizadas desde la farmacia comunitaria}

Las intervenciones más frecuentes, en los documentos revisados fueron la revisión de la medicación a domicilio, en 22 de ellos (11,12,15-17,20,23,24,26,29-31,35,37,38,40,42,44,46,49,52,53). En 20 artículos la acción realizada consistió en las recomendaciones individualizadas al paciente y educación sobre el correcto uso manejo y seguridad de los medicamentos y/o dispositivos de medida $(10,13,17,18,21,24,30,31,32,33,34,35,37,39,41-43,45-48,51-53)$ y en 18 se identificaron los pro- 
blemas relacionados con la medicación (PRM) (11,13-15,19,21,24-26,30,31,35,36,47,49-52). En 13 trabajos la intervención realizada por el farmacéutico comunitario permitió identificar problemas de falta de adherencia al tratamiento $(15,22,24,34,41-43,45,47-49,51,53)$. 4 intervenciones, fueron recomendaciones sobre la conservación y almacenaje de medicamentos o botiquines $(11,27,42,52)$.

\section{Resultados observados en los pacientes que recibieron la atención de la salud a domicilio}

Entre las consecuencias derivadas de las intervenciones realizadas desde la farmacia comunitaria, en 19 artículos se destacó la detección y/o resolución de situaciones de falta de adherencia $(10,11,14,17,19-22,27,29,32-34,42,44,46,47,52,53)$.

También, 17 trabajos mostraban como las intervenciones farmacéuticas domiciliarias permitieron detectar carencias de información y/o mejorar el conocimiento del paciente sobre el proceso de uso de los medicamentos (administración, seguridad, dosis, posología) $(11,12,15,17,20,29,30,32$,34$38,40,49,50,53)$. Asimismo, en 13 estudios se consiguió la detección, prevención y/o la resolución de los PRM $(10,12,19,21,24-26,30,31,44,46,47,49)$.

En relación a los resultados en salud tras las intervenciones farmacéuticas, en 7 trabajos revisados se observó un mejor uso de los medicamentos $(20,24,27,31,37,50,52)$ y en 6 artículos, se consiguió una mejoría en los síntomas y resultados clínicos en salud (10,16,24,43-45). En 2 estudios se observó una sustancial mejora en el almacenaje de los medicamentos y botiquines $(27,52)$.

Otros resultados, relacionados con la intervención desde la farmacia comunitaria fueron: el ahorro de costes que supuso la atención farmacéutica a domicilio, en 5 artículos $(14,16,21,36,44)$ y la reducción de los ingresos hospitalarios, en otros 5 trabajos $(21,22,36,47,51) ; 5$ artículos informaron que no había dicha reducción y, en ocasiones, incluso se observó un aumento en los ingresos hospitalarios $(25,35,39,41,51)$. En 9 trabajos los pacientes aceptaron las recomendaciones hechas por el farmacéutico $(15,17,18,23,35,36,43,47,50)$. 


\begin{tabular}{|c|c|c|c|c|c|c|c|}
\hline Autor, año & Diseño & Población estudiada & Patología & País & Periodo del estudio & Tipo de intervención & Resultado observado \\
\hline $\begin{array}{l}\text { Daliri et al. } \\
2019(10)\end{array}$ & $\begin{array}{l}\text { Estudio prospectivo } \\
\text { multicéntrico }\end{array}$ & $\begin{array}{l}N=456 \\
\text { Gl: } n=222 \\
H / M=124 / 98 \\
E d a d=70,2 \pm 12,8 \\
G C: n=234 \\
H / M=130 / 104 \\
E d a d=70,8 \pm 11,9\end{array}$ & $\begin{array}{l}\text { Pacientes ingresados } \\
\text { en el hospital con } \\
\text { medicación crónica }\end{array}$ & Países Bajos & $\begin{array}{l}\text { Periodo total: } 01 / 2016 \text { al } \\
11 / 2016 \\
\text { Periodo de reclutamiento: } \\
01 / 2016 \text { al } 06 / 2016 \\
\text { Periodo de seguimiento: } \\
4 \text { semanas }\end{array}$ & $\begin{array}{l}\text { Se adiestró a los pacientes el correcto uso } \\
\text { de los medicamentos con una atención } \\
\text { de seguimiento post-hospitalización en el } \\
\text { hogar. }\end{array}$ & $\begin{array}{l}\text { Los pacientes tienen menos síntomas, } \\
\text { recuerdan más la medicación y su } \\
\text { continuidad (mejor adherencia). Los } \\
\text { farmacéuticos identifican, resuelven y } \\
\text { previenen PRM. }\end{array}$ \\
\hline $\begin{array}{l}\text { Papastergiou } \\
\text { et al. } \\
2019 \text { (11) }\end{array}$ & $\begin{array}{l}\text { Estudio descriptivo } \\
\text { transversal }\end{array}$ & $\begin{array}{l}N=100 \\
H / M=42 / 58 \\
E d a d=76,9\end{array}$ & $\begin{array}{l}\text { Polimedicados } \\
\text { crónicos }\end{array}$ & EE. UU. & $\begin{array}{l}\text { Periodo de reclutamiento: } \\
\text { 01/2016 al 03/2017 }\end{array}$ & $\begin{array}{l}\text { Se hizo una revisión de medicamentos, } \\
\text { se identificaron PRM reales y potenciales, } \\
\text { se recomendó un uso y unas prácticas de } \\
\text { organización/ almacenamiento de estos en } \\
\text { el hogar. }\end{array}$ & $\begin{array}{l}\text { La revisión de la medicación a domicilio } \\
\text { podría minimizar el uso inapropiado de la } \\
\text { medicación y maximizar el ahorro en los } \\
\text { costes en salud. }\end{array}$ \\
\hline $\begin{array}{l}\text { Fujita et al. } \\
2019 \text { (12) }\end{array}$ & Grupos focales & $\begin{array}{l}\mathrm{N}=24 \\
\mathrm{H} / \mathrm{M}=\mathrm{No} \text { consta } \\
\mathrm{Edad}=\text { No consta }\end{array}$ & $\begin{array}{l}\text { Ancianos con poca } \\
\text { movilidad }\end{array}$ & Japón & $\begin{array}{l}\text { Periodo de reclutamiento: } \\
\text { 12/2016 al 01/2017 }\end{array}$ & $\begin{array}{l}\text { Se dio un suministro de medicamentos y } \\
\text { una revisión de estos a domicilio. }\end{array}$ & $\begin{array}{l}\text { La formación impartida facilitó el } \\
\text { conocimiento de su tratamiento y la } \\
\text { reducción de los PRM. }\end{array}$ \\
\hline $\begin{array}{l}\text { Toivo et al. } \\
2019 \text { (13) }\end{array}$ & $\begin{array}{l}\text { Ensayo aleatorio } \\
\text { controlado }\end{array}$ & $\begin{array}{l}N=129 \\
\mathrm{Gl}: \mathrm{n}=65 \\
\mathrm{H} / \mathrm{M}=21 / 44 \\
\mathrm{Edad}=81,6 \pm 7,1 \\
\mathrm{GC}: \mathrm{n}=64 \\
\mathrm{H} / \mathrm{M}=18 / 46 \\
\mathrm{Edad}=84,0 \pm 6,8\end{array}$ & Ancianos & Finlandia & $\begin{array}{l}\text { Periodo total: } 09 / 2015 \text { al } \\
12 / 2016 \\
\text { Periodo de reclutamiento: } \\
\text { 09/2015 al } 12 / 2015 \\
\text { Periodo de seguimiento: } \\
12 \text { meses }\end{array}$ & $\begin{array}{l}\text { Los farmacéuticos a domicilio } \\
\text { informaron sobre el correcto uso de los } \\
\text { medicamentos, hicieron una reconciliación } \\
\text { de estos, identificaron PRM e hicieron } \\
\text { recomendaciones para resolverlos. }\end{array}$ & $\begin{array}{l}\text { Tras la revisión exhaustiva de la } \\
\text { medicación y un seguimiento adecuado, } \\
\text { se hizo un ajuste de los tratamientos y de } \\
\text { las pruebas clínica asociadas. }\end{array}$ \\
\hline $\begin{array}{l}\text { Kayyali et al. } \\
2019(14)\end{array}$ & $\begin{array}{l}\text { Estudio descriptivo } \\
\text { transversal }\end{array}$ & $\begin{array}{l}N=133 \\
H / M=39 / 82 \\
12 \sin \text { información } \\
\text { Edad }=83\end{array}$ & $\begin{array}{l}\text { Ancianos } \\
\text { polimedicados en sus } \\
\text { domicilios }\end{array}$ & Reino Unido & $\begin{array}{l}\text { Periodo de seguimiento: } \\
\text { 05/2015 al 01/2016 }\end{array}$ & $\begin{array}{l}\text { Revisión de medicación a domicilio, } \\
\text { se identificaron PRM, problemas de } \\
\text { adherencia monitorización y un control } \\
\text { general de la situación. }\end{array}$ & $\begin{array}{l}\text { Las visitas domiciliarias de los } \\
\text { farmacéuticos pueden ayudar a identificar } \\
\text { las diversas necesidades de atención de } \\
\text { pacientes aislados confinados en su la } \\
\text { casa y ayudar a integrar sus requisitos de } \\
\text { atención }\end{array}$ \\
\hline $\begin{array}{l}\text { Corsi et al. } \\
2018 \text { (15) }\end{array}$ & $\begin{array}{l}\text { Programa piloto } \\
\text { prospectivo }\end{array}$ & $\begin{array}{l}\mathrm{N}=25 \\
\mathrm{H} / \mathrm{M}=12 / 13 \\
\mathrm{Edad}=79,88 \pm 8,08\end{array}$ & $\begin{array}{l}\text { Ancianos } \\
\text { polimedicados } \\
\text { crónicos }\end{array}$ & EE. UU. & $\begin{array}{l}\text { Periodo de recopilación de } \\
\text { datos: } \\
\text { 01/2017 al 05/2017 }\end{array}$ & $\begin{array}{l}\text { Hubo una gestión de medicamentos } \\
\text { para identificar pacientes comórbidos, } \\
\text { polimedicados, PRM y se creó un plan de } \\
\text { acción de medicación en el hogar. }\end{array}$ & $\begin{array}{l}\text { Se logró un ahorro de costes, reducción } \\
\text { PRM y mejor control por parte de los } \\
\text { pacientes de su medicación. }\end{array}$ \\
\hline $\begin{array}{l}\text { Clifton et al. } \\
2018(16)\end{array}$ & $\begin{array}{l}\text { Análisis } \\
\text { retrospectivo }\end{array}$ & $\begin{array}{l}\mathrm{N}=341 \\
\mathrm{H} / \mathrm{M}=\text { No consta } \\
\mathrm{Edad}=62,4\end{array}$ & $\begin{array}{l}\text { Polimedicados } \\
\text { crónicos }\end{array}$ & EE. UU. & $\begin{array}{l}\text { Periodo de reclutamiento: } \\
\text { 09/2014 al } 12 / 2016 \\
\text { Periodo de seguimiento: } \\
12 \text { meses }\end{array}$ & $\begin{array}{l}\text { Se mejoraron los servicios farmacéuticos } \\
\text { con visitas domiciliarias y revisiones de la } \\
\text { medicación. }\end{array}$ & $\begin{array}{l}\text { Se consiguió una reducción de gastos y } \\
\text { mejoras en los resultados de la salud. }\end{array}$ \\
\hline $\begin{array}{l}\text { Walus et al. } \\
2017 \text { (17) }\end{array}$ & Estudio piloto & $\begin{array}{l}\mathrm{N}=122 \\
\mathrm{H} / \mathrm{M}=45 / 77 \\
\mathrm{Edad}=71\end{array}$ & $\begin{array}{l}\text { Desorden } \\
\text { cardiovascular, } \\
\text { trastorno de salud } \\
\text { mental, desorden } \\
\text { endocrino y alergias. }\end{array}$ & EE. UU. & $\begin{array}{l}\text { Periodo total: 05/2015 al } \\
\text { 07/2016 }\end{array}$ & $\begin{array}{l}\text { Prestación de servicios de farmacia a } \\
\text { domicilio (revisión de medicamentos } \\
\text { y educación a los pacientes sobre el } \\
\text { medicamento) }\end{array}$ & $\begin{array}{l}\text { Se mejoro la educación para el paciente } \\
\text { y se aumentó la adherencia. Hubo una } \\
\text { necesidad de mejorar los servicios de } \\
\text { farmacia a domicilio porque los pacientes } \\
\text { preferían las consultas telefónicas }\end{array}$ \\
\hline $\begin{array}{l}\text { Elliott et al. } \\
2017 \text { (18) }\end{array}$ & $\begin{array}{l}\text { Estudio teórico } \\
\text { de co-creación y } \\
\text { acción participativa }\end{array}$ & $\begin{array}{l}\mathrm{N}=1089 \\
\mathrm{H} / \mathrm{M}=\text { No consta } \\
\mathrm{Edad}=\text { No consta }\end{array}$ & $\begin{array}{l}\text { Pacientes con riesgos } \\
\text { RAM, polimedicados y } \\
\text { ancianos. }\end{array}$ & Australia & $\begin{array}{l}\text { Periodo total: } \\
\text { 09/2014 al 11/2015 } \\
\text { Periodo de seguimiento: } \\
15 \text { meses }\end{array}$ & $\begin{array}{l}\text { Se creó un modelo colaborativo entre un } \\
\text { servicio de enfermería y un farmacéutico } \\
\text { a domicilio, para mejorar el manejo del } \\
\text { medicamento y su seguridad. }\end{array}$ & $\begin{array}{l}\text { El modelo de colaboración farmacéutico- } \\
\text { enfermería permitió resolver problemas } \\
\text { relacionados con la medicación. La mejora } \\
\text { del asesoramiento redujo los RAM. }\end{array}$ \\
\hline $\begin{array}{l}\text { Ong et al. } \\
2017 \text { (19) }\end{array}$ & $\begin{array}{l}\text { Estudio } \\
\text { observacional } \\
\text { retrospectivo }\end{array}$ & $\begin{array}{l}N=107 \\
H / M=52 / 55 \\
E d a d=75,6 \pm 7,6\end{array}$ & Polimedicados & Singapur & $\begin{array}{l}\text { Periodo de reclutamiento: } \\
\text { 03/2011 al } 12 / 2012 \\
\text { Periodo de seguimiento: } \\
1 \text { mes }\end{array}$ & $\begin{array}{l}\text { Asesoramiento farmacológico, } \\
\text { identificación de PRM y recomendaciones } \\
\text { en el hogar, }\end{array}$ & $\begin{array}{l}\text { Reducción del riesgo de ingreso } \\
\text { hospitalario y reducción del coste. } \\
\text { Se resolvieron los PRM y se mejoró la } \\
\text { adherencia. }\end{array}$ \\
\hline $\begin{array}{l}\text { Basheti et al. } \\
2016(20)\end{array}$ & $\begin{array}{l}\text { Ensayo aleatorio } \\
\text { controlado }\end{array}$ & $\begin{array}{l}N=97 \\
\text { Gl: } n=48 \\
H / M=17 / 31 \\
\text { Edad }=63,13 \pm 7,99 \\
G C: n=49 \\
\text { H/M= }=12 / 37 \\
\text { Edad }=58,39 \pm 12,8\end{array}$ & $\begin{array}{l}\text { Enfermedades } \\
\text { crónicas }\end{array}$ & Jordania & $\begin{array}{l}\text { Periodo total: } \\
\text { 06/2014 al 12/2014 } \\
\text { Periodo de seguimiento: } \\
3 \text { meses }\end{array}$ & $\begin{array}{l}\text { Identificación, en el hogar, de PRM, tipo y } \\
\text { frecuencia, además de su resolución por el } \\
\text { farmacéutico en conjunto con el médico. }\end{array}$ & $\begin{array}{l}\text { Hubo una reducción de PRM y una mejor } \\
\text { adherencia al medicamento }\end{array}$ \\
\hline $\begin{array}{l}\text { Steele et al. } \\
2016 \text { (21) }\end{array}$ & $\begin{array}{l}\text { Estudio de pre y } \\
\text { post-intervención }\end{array}$ & $\begin{array}{l}\mathrm{N}=25 \\
\mathrm{H} / \mathrm{M}=7 / 18 \\
\mathrm{Edad}=88\end{array}$ & $\begin{array}{l}\text { Ancianos geriátricos } \\
\text { con polimedicación }\end{array}$ & EE. UU. & $\begin{array}{l}\text { Periodo de reclutamiento: } \\
\text { 09/2014 al 01/2015 }\end{array}$ & $\begin{array}{l}\text { Se evaluó los efectos de las revisiones } \\
\text { integrales de medicamentos a domicilio. }\end{array}$ & $\begin{array}{l}\text { Se mejoró la adherencia y administración } \\
\text { de medicamentos y se redujeron las } \\
\text { técnicas incorrectas con respecto a ellos. }\end{array}$ \\
\hline $\begin{array}{l}\text { Kalista et al. } \\
2015 \text { (22) }\end{array}$ & $\begin{array}{l}\text { Proyecto piloto } \\
\text { prospectivo }\end{array}$ & $\begin{array}{l}N=10 \\
H / M=4 / 6 \\
E d a d=81,3 \pm 7,3\end{array}$ & Insuficiencia cardiaca & EE. UU. & $\begin{array}{l}\text { Periodo de reclutamiento: } \\
\text { 12/2013 al 04/2014 } \\
\text { Periodo de seguimiento: } \\
5 \text { semanas }\end{array}$ & $\begin{array}{l}\text { Atención domiciliaria para mejorar la } \\
\text { adherencia. }\end{array}$ & $\begin{array}{l}\text { Se mejoró la adherencia y se redujo las } \\
\text { tasas de reingresos en el hospital. }\end{array}$ \\
\hline $\begin{array}{l}\text { Carter et al. } \\
2015 \text { (23) }\end{array}$ & $\begin{array}{l}\text { Estudio descriptivo } \\
\text { transversal }\end{array}$ & $\begin{array}{l}N=595 \\
H / M=298 / 297 \\
E d a d=65\end{array}$ & $\begin{array}{l}\text { Pacientes que han } \\
\text { experimentado } \\
\text { una revisión de los } \\
\text { medicamentos en el } \\
\text { hogar }\end{array}$ & Australia & $\begin{array}{l}\text { Periodo de recopilación de } \\
\text { datos: } \\
\text { 11/2008 al 05/2009 }\end{array}$ & $\begin{array}{l}\text { Cuestionario para evaluar la capacidad } \\
\text { de escucha, de comunicación y de la } \\
\text { efectividad de la revisión de medicamentos } \\
\text { a domicilio. }\end{array}$ & $\begin{array}{l}\text { Los farmacéuticos tienen una buena } \\
\text { habilidad para escuchar y una eficacia en } \\
\text { la comunicación. }\end{array}$ \\
\hline $\begin{array}{l}\text { Pherson et al. } \\
2014 \text { (24) }\end{array}$ & $\begin{array}{l}\text { Estudio descriptivo } \\
\text { transversal }\end{array}$ & $\begin{array}{l}\mathrm{N}=50 \\
\mathrm{H} / \mathrm{M}=23 / 27 \\
\mathrm{Edad}=60 \pm 12,6\end{array}$ & $\begin{array}{l}\text { Ingresados con alta } \\
\text { medica }\end{array}$ & EE. UU. & $\begin{array}{l}\text { Periodo de reclutamiento: } \\
\text { 02/2012 al 09/2013 }\end{array}$ & $\begin{array}{l}\text { Se ofreció un servicio de gestión de } \\
\text { medicamento a domicilio para educar } \\
\text { sobre el correcto uso de medicamentos, } \\
\text { para mejorar la adherencia y para evaluar } \\
\text { y crear conciencia de PRM. También se } \\
\text { revisaron los medicamentos. }\end{array}$ & $\begin{array}{l}\text { Mejora del paciente al administrar su } \\
\text { medicación, evitaron PRM, hubo una } \\
\text { mejora de la automedicación. }\end{array}$ \\
\hline $\begin{array}{l}\text { Reidt et al. } \\
2014 \text { (25) }\end{array}$ & Estudio prospectivo & $\begin{array}{l}N=533 \\
\text { Gi: } n=153 \\
H / M=45 / 108 \\
E d a d=18-65 \\
\text { Gc: } n=380 \\
H / M=132 / 248 \\
\text { Edad }=18-65\end{array}$ & Polimedicados & EE. UU. & $\begin{array}{l}\text { Periodo de reclutamiento: } \\
\text { 01/2009 al } 12 / 2010 \\
\text { Periodo de seguimiento: } \\
60 \text { días }\end{array}$ & $\begin{array}{l}\text { Modelo de atención medica domiciliaria } \\
\text { con un farmacéutico para los ajustes de } \\
\text { dosis, interrupción de medicamentos, } \\
\text { monitoreo y para detectar y prevenir PRM } \\
\text { y RAM. }\end{array}$ & $\begin{array}{l}\text { No hubo diferencias significativas en los } \\
\text { reingresos hospitalarios. Se identificaron } \\
\text { más PRM y antes. }\end{array}$ \\
\hline $\begin{array}{l}\text { Kogut et al. } \\
2014(26)\end{array}$ & $\begin{array}{l}\text { Estudio descriptivo } \\
\text { transversal }\end{array}$ & $\begin{array}{l}\mathrm{N}=30 \\
\mathrm{H} / \mathrm{M}=16 / 14 \\
\mathrm{Edad}=65\end{array}$ & $\begin{array}{l}\text { Enfermedades } \\
\text { crónicas }\end{array}$ & EE. UU. & $\begin{array}{l}\text { Periodo de seguimiento: } \\
\text { 08/2011 al 02/2012 }\end{array}$ & $\begin{array}{l}\text { Revisión de la medicación para identificar } \\
\text { PRM y utilización de un sistema electrónico } \\
\text { de registro de salud personal en el hogar. }\end{array}$ & $\begin{array}{l}\text { Se identificó muchos PRM y se } \\
\text { solucionaron. El sistema electrónico fue } \\
\text { eficaz pero no hubo suficiente muestra } \\
\text { para sacar conclusiones. }\end{array}$ \\
\hline $\begin{array}{l}\text { Kalyango et al. } \\
2012(27)\end{array}$ & $\begin{array}{l}\text { Estudio descriptivo } \\
\text { transversal }\end{array}$ & $\begin{array}{l}\mathrm{N}=207 \\
\mathrm{H} / \mathrm{M}=70 / 137 \\
\mathrm{Edad}=49 \pm 17,1\end{array}$ & $\begin{array}{l}\text { Enfermedades } \\
\text { crónicas, artritis, } \\
\text { tiroides, diabetes... }\end{array}$ & Uganda & $\begin{array}{l}\text { Periodo total: 06/2010 al } \\
07 / 2010\end{array}$ & $\begin{array}{l}\text { Visita a domicilio para hacer unas } \\
\text { entrevistas e informar sobre el correcto } \\
\text { almacenamiento de medicamentos. }\end{array}$ & $\begin{array}{l}\text { Se mejoró el almacenamiento de } \\
\text { medicamentos, se les administro un horario } \\
\text { para su administración y se informó de la } \\
\text { práctica inadecuada de estos. }\end{array}$ \\
\hline $\begin{array}{l}\text { El Hajj et al. } \\
2012(28)\end{array}$ & $\begin{array}{l}\text { Estudio descriptivo } \\
\text { transversal }\end{array}$ & $\begin{array}{l}N=297 \\
H / M=199 / 98 \\
E d a d=18-50\end{array}$ & Ninguna & Qatar & $\begin{array}{l}\text { Periodo total: } \\
5 \text { meses }\end{array}$ & $\begin{array}{l}\text { Opiniones del público por realizar pruebas } \\
\text { diagnósticas en casa por farmacéuticos. }\end{array}$ & $\begin{array}{l}\text { Se observó poca actitud e interés por parte } \\
\text { de los farmacéuticos para realizar pruebas } \\
\text { diagnósticas. }\end{array}$ \\
\hline
\end{tabular}




\begin{tabular}{|c|c|c|c|c|c|c|c|}
\hline Autor, año & Diseño & Población estudiada & Patología & País & Periodo del estudio & Tipo de intervención & Resultado observado \\
\hline $\begin{array}{l}\text { Kwint et al. } \\
2012 \text { (29) }\end{array}$ & $\begin{array}{l}\text { Estudio descriptivo } \\
\text { transversal }\end{array}$ & $\begin{array}{l}\mathrm{N}=155 \\
\mathrm{H} / \mathrm{M}=71 / 84 \\
\mathrm{Edad}=76\end{array}$ & $\begin{array}{l}\text { Ancianos } \\
\text { polimedicados y que } \\
\text { usan cardiovasculares } \\
\text { o antidiabéticos }\end{array}$ & Países Bajos & $\begin{array}{l}\text { Periodo de reclutamiento: } \\
\text { 02/2008 al 08/2010 } \\
\text { Periodo de seguimiento: } \\
12 \text { meses }\end{array}$ & $\begin{array}{l}\text { Revisiones para reducir los PRM, controlar } \\
\text { el colesterol y la HTA en el hogar. }\end{array}$ & $\begin{array}{l}\text { La intervención permitió la identificación de } \\
\text { PRM y hubo un mayor y mejor control de la } \\
\text { medicación. }\end{array}$ \\
\hline $\begin{array}{l}\text { Lee et al. } \\
2012(30)\end{array}$ & $\begin{array}{l}\text { Estudio de grupos } \\
\text { focales }\end{array}$ & $\begin{array}{l}\mathrm{N}=32 \\
\mathrm{H} / \mathrm{M}=17 / 15 \\
\mathrm{Edad}=65-86\end{array}$ & Polimedicados & Australia & $\begin{array}{l}\text { Periodo de reclutamiento: } \\
\text { 12/2009 al 08/2010 }\end{array}$ & Revisión medicamentos en aldeas. & $\begin{array}{l}\text { Hubo problemas para captar pacientes } \\
\text { que quisieran un servicio de revisión } \\
\text { de medicamentos, pero mejoró el } \\
\text { conocimiento y la adherencia de } \\
\text { medicamentos. }\end{array}$ \\
\hline $\begin{array}{l}\text { Elliott et al. } \\
2012(31)\end{array}$ & $\begin{array}{l}\text { Estudio comparativo } \\
\text { aleatorizado }\end{array}$ & $\begin{array}{l}N=80 \\
\text { Gi: } n=40 \\
H / M=14 / 26 \\
E d a d=85,5 \\
\text { Gc: } n=40 \\
H / M=15 / 25 \\
E d a d=83,0\end{array}$ & Ancianos & Australia & $\begin{array}{l}\text { Periodo de reclutamiento: } \\
\text { 08/2009 al 04/2010 } \\
\text { Periodo de seguimiento: } \\
20 \text { semanas }\end{array}$ & $\begin{array}{l}\text { Revisión integral a domicilio de } \\
\text { medicamentos, detección de PRM y } \\
\text { recomendaciones. }\end{array}$ & $\begin{array}{l}\text { Se detectó PRM, se eliminó los } \\
\text { medicamentos sobrantes y se dio } \\
\text { educación sobre los medicamentos. }\end{array}$ \\
\hline $\begin{array}{l}\text { Fikri- } \\
\text { Benbrahim } \\
\text { et al. } \\
2012 \text { (32) }\end{array}$ & $\begin{array}{l}\text { Estudio quasi- } \\
\text { experimental }\end{array}$ & $\begin{array}{l}\mathrm{N}=176 \\
\mathrm{H} / \mathrm{M}=66 / 110 \\
\mathrm{Edad}=62,0 \pm 11,6\end{array}$ & Hipertensión arterial & España & $\begin{array}{l}\text { Periodo de reclutamiento: } \\
1 \text { mes } \\
\text { Periodo de seguimiento: } \\
20 \text { semanas }\end{array}$ & $\begin{array}{l}\text { Monitoreo de hipertensión arterial en el } \\
\text { hogar. }\end{array}$ & $\begin{array}{l}\text { Aumentó la adherencia y mejoró el control } \\
\text { de la presión arterial. }\end{array}$ \\
\hline $\begin{array}{l}\text { Stafford et al. } \\
2012(33)\end{array}$ & $\begin{array}{l}\text { Ensayo de cohorte } \\
\text { prospectivo no } \\
\text { aleatorizado y } \\
\text { controlado }\end{array}$ & $\begin{array}{l}\mathrm{N}=268 \\
\mathrm{Gl}: \mathrm{n}=129 \\
\mathrm{H} / \mathrm{M}=77 / 52 \\
\mathrm{Edad}=67,7 \\
\mathrm{GC}: \mathrm{n}=139 \\
\mathrm{H} / \mathrm{M}=83 / 56 \\
\mathrm{Edad}=66,2\end{array}$ & $\begin{array}{l}\text { Pacientes tratados } \\
\text { con Warfarina }\end{array}$ & Australia & $\begin{array}{l}\text { Periodo de reclutamiento: } \\
11 / 2008 \text { al } 12 / 2009\end{array}$ & $\begin{array}{l}\text { El paciente recibió instrucciones a } \\
\text { domicilio por el farmacéutico para la } \\
\text { Warfarina después del alta. }\end{array}$ & $\begin{array}{l}\text { Se creó un mejor conocimiento de la } \\
\text { Warfarina y mejoró la terapia con ella. }\end{array}$ \\
\hline $\begin{array}{l}\text { Sheridan et al. } \\
2012 \text { (34) }\end{array}$ & Estudio piloto & $\begin{array}{l}\mathrm{N}=27 \\
\mathrm{H} / \mathrm{M}=8 / 19 \\
\mathrm{Edad}=75\end{array}$ & Polimedicados & Nueva Zelanda & $\begin{array}{l}\text { Periodo de reclutamiento: } \\
\text { 10/2007 al 09/2008 } \\
\text { Periodo de seguimiento: } \\
12 \text { meses }\end{array}$ & $\begin{array}{l}\text { Análisis de una revisión del uso de } \\
\text { medicamentos a domicilio, su conocimiento } \\
\text { y adherencia }\end{array}$ & $\begin{array}{l}\text { Los pacientes dijeron tener mayor } \\
\text { conocimiento y adherencia, aunque la } \\
\text { captación de pacientes fue complicada }\end{array}$ \\
\hline $\begin{array}{l}\text { Roughead } \\
\text { et al. } \\
2011 \text { (35) }\end{array}$ & $\begin{array}{l}\text { Estudio de cohorte } \\
\text { retrospectivo }\end{array}$ & $\begin{array}{l}N=17136 \\
\text { Gi: } n=816 \\
H / M=64 / 752 \\
E d a d=81,6 \\
G c: n=16320 \\
H / M=65 / 16255 \\
E d a d=81,4\end{array}$ & $\begin{array}{l}\text { Veteranos de guerra } \\
\text { con Warfarina }\end{array}$ & Australia & $\begin{array}{l}\text { Periodo total: } \\
\text { 01/2004 al 07/2006 } \\
\text { Periodo de seguimiento: } \\
12 \text { meses }\end{array}$ & $\begin{array}{l}\text { Visita a domicilio cada 2-6 meses para } \\
\text { una educación de uso de medicamentos, } \\
\text { detección de PRM y una revisión } \\
\text { exhaustiva de la receta médica. }\end{array}$ & $\begin{array}{l}\text { Se aumento la hospitalización por la } \\
\text { mayor información de RAM. Se mejoró la } \\
\text { oportunidad de enseñar y aconsejar al } \\
\text { paciente. }\end{array}$ \\
\hline $\begin{array}{l}\text { Flanagan et al. } \\
2010(36)\end{array}$ & Estudio de cohorte & $\begin{array}{l}\mathrm{N}=836 \\
\mathrm{H} / \mathrm{M}=333 / 503 \\
\mathrm{Edad}=80,3\end{array}$ & $\begin{array}{l}\text { Ancianos } \\
\text { polimedicados }\end{array}$ & EE. UU. & $\begin{array}{l}\text { Periodo total: 04/2005 al } \\
03 / 2007\end{array}$ & $\begin{array}{l}\text { Programa de administración de } \\
\text { medicamentos a domicilio para evaluar el } \\
\text { régimen de medicamentos para identificar } \\
\text { y resolver PRM. }\end{array}$ & $\begin{array}{l}\text { Se usó menos los recursos de salud, } \\
\text { se redujeron los costes y se aceptaron } \\
\text { las recomendaciones para mejorar la } \\
\text { idoneidad de la medicación }\end{array}$ \\
\hline $\begin{array}{l}\text { Hugtenburg } \\
\text { et al. } \\
2009 \text { (37) }\end{array}$ & $\begin{array}{l}\text { Estudio de } \\
\text { intervención } \\
\text { controlada }\end{array}$ & $\begin{array}{l}N=715 \\
\text { Gi: } n=336 \\
\text { H/M }=164 / 172 \\
\text { Edad }=69,7 \pm 15,0 \\
\text { Gc: } n=379 \\
\text { H/M }=177 / 202 \\
\text { Edad }=72,7 \pm 11,2\end{array}$ & Polimedicados & Países Bajos & $\begin{array}{l}\text { Periodo de reclutamiento: } 2001 \\
\text { al } 2003 \\
\text { Periodo de seguimiento: } \\
9 \text { meses }\end{array}$ & $\begin{array}{l}\text { Desarrollo de un protocolo de intervención } \\
\text { de medicamentos a domicilio para } \\
\text { revisarlos y dar información sobre su } \\
\text { correcto uso. }\end{array}$ & $\begin{array}{l}\text { El protocolo creó un control de } \\
\text { medicamentos dispensados y mejoró la } \\
\text { información recibida a los pacientes, pero } \\
\text { hubo una tasa alta de baja adherencia en } \\
\text { pacientes crónicos. }\end{array}$ \\
\hline $\begin{array}{l}\text { Moultry et al. } \\
2008 \text { (38) }\end{array}$ & $\begin{array}{l}\text { Estudio descriptivo } \\
\text { transversal }\end{array}$ & $\begin{array}{l}\mathrm{N}=18 \\
\mathrm{H} / \mathrm{M}=7 / 11 \\
\mathrm{Edad}=71-80\end{array}$ & Polimedicados & EE. UU. & $\begin{array}{l}\text { Periodo de reclutamiento: } \\
\text { 05/2006 al 05/2007 }\end{array}$ & $\begin{array}{l}\text { Encuesta a participantes después de la } \\
\text { terapia de gestión de medicamentos en el } \\
\text { hogar y de una revisión de medicamentos. }\end{array}$ & $\begin{array}{l}\text { Hubo una falta de conocimientos en los } \\
\text { farmacéuticos, pero obtuvieron éxito en la } \\
\text { terapia de gestión de medicamentos y de } \\
\text { la información a los pacientes. }\end{array}$ \\
\hline $\begin{array}{l}\text { Holland et al. } \\
2007 \text { (39) }\end{array}$ & $\begin{array}{l}\text { Ensayo aleatorio } \\
\text { controlado }\end{array}$ & $\begin{array}{l}N=291 \\
G l: n=148 \\
H / M=94 / 54 \\
E d a d=77,6 \pm 9,0 \\
G C: n=143 \\
H / M=90 / 53 \\
E d a d=76,4 \pm 9,5\end{array}$ & Insuficiencia cardiaca & Reino Unido & $\begin{array}{l}\text { Periodo de reclutamiento: } \\
12 / 2003 \text { al } 03 / 2005 \\
\text { Periodo de seguimiento: } \\
6 \text { meses }\end{array}$ & $\begin{array}{l}\text { Visita domiciliaria de farmacéuticos para } \\
\text { dar información sobre la insuficiencia } \\
\text { cardiaca, su medicación, dieta y ejercicio. } \\
\text { Se creó unas tarjetas de monitoreo diario } \\
\text { de signos y síntomas. }\end{array}$ & $\begin{array}{l}\text { No hubo reducción en los ingresos } \\
\text { hospitalarios ni tubo efecto sobre la } \\
\text { mortalidad. }\end{array}$ \\
\hline $\begin{array}{l}\text { Salter et al. } \\
2007 \text { (40) }\end{array}$ & Análisis discursivo & $\begin{array}{l}\mathrm{N}=29 \\
\mathrm{H} / \mathrm{M}=11 / 18 \\
\mathrm{Edad}=83,3\end{array}$ & $\begin{array}{l}\text { Polimedicados } \\
\text { ingresados } \\
\text { previamente en } \\
\text { hospital }\end{array}$ & Reino Unido & $\begin{array}{l}\text { Periodo de reclutamiento: } \\
\text { 10/2002 al 12/2002 }\end{array}$ & Revisión de medicamentos a domicilio. & $\begin{array}{l}\text { La información facilitada por los } \\
\text { farmacéuticos no siempre fue aceptada. } \\
\text { Por el contrario, la información e } \\
\text { instrucciones sobre los medicamentos sí. }\end{array}$ \\
\hline $\begin{array}{l}\text { Triller et al. } \\
2007 \text { (41) }\end{array}$ & $\begin{array}{l}\text { Ensayo aleatorio } \\
\text { controlado }\end{array}$ & $\begin{array}{l}N=154 \\
\text { Gi: } n=77 \\
H / M=21 / 56 \\
E d a d=81,3 \pm 9,3 \\
\text { Gc: } n=77 \\
H / M=22 / 55 \\
\text { Edad }=78,1 \pm 11,2\end{array}$ & Insuficiencia cardiaca & EE. UU. & $\begin{array}{l}\text { Periodo de reclutamiento: } \\
\text { 06/2002 al 05/2004 } \\
\text { Periodo de seguimiento: } \\
6 \text { meses }\end{array}$ & $\begin{array}{l}\text { A los pacientes se les dio en sus casas } \\
\text { sugerencias de cambios de hábitos como } \\
\text { fumar y una dieta equilibrada. Se les ayudó } \\
\text { con la adherencia y con el uso inapropiado } \\
\text { de medicamentos. }\end{array}$ & $\begin{array}{l}\text { No hubo diferencias significativas entre } \\
\text { el grupo de intervención y el grupo } \\
\text { control con respecto a las muertes y a la } \\
\text { hospitalización. }\end{array}$ \\
\hline $\begin{array}{l}\text { Sorensen et al. } \\
2006(42)\end{array}$ & $\begin{array}{l}\text { Estudio descriptivo } \\
\text { transversal }\end{array}$ & $\begin{array}{l}N=204 \\
H / M=144 / 60 \\
E d a d=72\end{array}$ & $\begin{array}{l}\text { Polimedicados mal } \\
\text { controlados }\end{array}$ & Australia & No consta & $\begin{array}{l}\text { Revisión de medicamentos duplicados } \\
\text { y caducados, adherencia, creación de } \\
\text { un botiquín, creación de una rutina de de } \\
\text { medicación y educación sobre genéricos y } \\
\text { comerciales. }\end{array}$ & $\begin{array}{l}\text { Hubo una mejor adherencia a los } \\
\text { medicamentos, se eliminó el riesgo } \\
\text { de confusión con los medicamentos } \\
\text { caducados y el riesgo de duplicidad de } \\
\text { dosis. }\end{array}$ \\
\hline $\begin{array}{l}\text { Zillich et al. } \\
2005 \text { (43) }\end{array}$ & $\begin{array}{l}\text { Ensayo aleatorio } \\
\text { controlado }\end{array}$ & $\begin{array}{l}N=125 \\
\text { Gi: } n=64 \\
H / M=27 / 37 \\
E d a d=64,0 \pm 11,1 \\
\text { Gc: } n=61 \\
H / M=22 / 39 \\
E d a d=66,1 \pm 13,8\end{array}$ & Hipertensión & EE. UU. & $\begin{array}{l}\text { Periodo de seguimiento: } \\
3 \text { meses }\end{array}$ & $\begin{array}{l}\text { Se proporciono información educativa } \\
\text { sobre la enfermedad el uso de } \\
\text { medicamentos y su adherencia, se } \\
\text { recomendaron cambios en el estilo de vida } \\
\text { y se enseñó la medición de la tensión a } \\
\text { domicilio. }\end{array}$ & $\begin{array}{l}\text { Hubo una reducción de la presión arterial } \\
\text { tomada en el hogar y reducción de efectos } \\
\text { cardiovasculares. Hubo mucha aceptación } \\
\text { por parte de médicos y pacientes de los } \\
\text { consejos farmacéuticos. }\end{array}$ \\
\hline $\begin{array}{l}\text { Sorensen et al. } \\
2004(44)\end{array}$ & $\begin{array}{l}\text { Ensayo aleatorio } \\
\text { controlado de } \\
\text { efectividad }\end{array}$ & $\begin{array}{l}N=392 \\
\text { Gi: } 176 \\
H / M=113 / 63 \\
\text { Edad }=72,3 \\
G C: 216 \\
\text { H/M }=152 / 64 \\
\text { Edad }=71,4\end{array}$ & Polimedicados & Australia & $\begin{array}{l}\text { Periodo total: 02/1999 al } \\
\text { 05/2000 } \\
\text { Periodo de reclutamiento: } \\
\text { 08/1999 al 05/2000 } \\
\text { Periodo de seguimiento: } \\
8 \text { semanas }\end{array}$ & $\begin{array}{l}\text { Visitas a domicilio para revisar } \\
\text { la medicación con un equipo } \\
\text { multidisciplinario }\end{array}$ & $\begin{array}{l}\text { Hay mejoras en los resultados clínicos y los } \\
\text { costes. Se redujeron los PRM y se aumentó } \\
\text { la adherencia a la medicación. }\end{array}$ \\
\hline $\begin{array}{l}\text { Peterson et al. } \\
2004 \text { (45) }\end{array}$ & $\begin{array}{l}\text { Ensayo aleatorio } \\
\text { controlado } \\
\text { prospectivo }\end{array}$ & $\begin{array}{l}N=81 \\
\text { Gi: } n=39 \\
H / M=22 / 17 \\
E d a d=65,5 \pm 11,0 \\
\text { Gc: } n=42 \\
H / M=29 / 13 \\
E d a d=63,5 \pm 12,1\end{array}$ & Dislipemia & Australia & $\begin{array}{l}\text { Periodo de reclutamiento: } \\
\text { 04/2001 al 10/2001 } \\
\text { Periodo de seguimiento: } \\
6 \text { meses }\end{array}$ & $\begin{array}{l}\text { En los domicilios de los pacientes se } \\
\text { determinó el colesterol y se registró un } \\
\text { régimen de medicación. Se educó sobre } \\
\text { los objetivos y beneficios de la reducción } \\
\text { de lípidos y de la modificación del estilo } \\
\text { de vida }\end{array}$ & $\begin{array}{l}\text { Mejor control de la dislipemia y una } \\
\text { disminución significativa en el colesterol } \\
\text { total. }\end{array}$ \\
\hline
\end{tabular}




\begin{tabular}{|c|c|c|c|c|c|c|c|}
\hline Autor, año & Diseño & Población estudiada & Patología & País & Periodo del estudio & Tipo de intervención & Resultado observado \\
\hline $\begin{array}{l}\text { Triller et al. } \\
2003 \text { (46) }\end{array}$ & $\begin{array}{l}\text { Estudio descriptivo } \\
\text { transversal }\end{array}$ & $\begin{array}{l}\mathrm{N}=80 \\
\mathrm{H} / \mathrm{M}=\mathrm{No} \text { consta } \\
\mathrm{Edad}=\text { No consta }\end{array}$ & $\begin{array}{l}\text { Polimedicados } \\
\text { crónicos }\end{array}$ & EE. UU. & $\begin{array}{l}\text { Periodo de reclutamiento: } \\
07 / 2001 \text { al 03/2002 }\end{array}$ & $\begin{array}{l}\text { Revisión de medicamentos a domicilio, } \\
\text { se descartaron los caducados, se } \\
\text { educó sobre sus medicamentos y } \\
\text { su autoadministración. Se hicieron } \\
\text { recomendaciones para reducir PRM. }\end{array}$ & $\begin{array}{l}\text { Se pudo identificar y resolver PRM y hubo } \\
\text { una mejora del cumplimiento. }\end{array}$ \\
\hline $\begin{array}{l}\text { Naunton et al. } \\
2003(47)\end{array}$ & $\begin{array}{l}\text { Estudio aleatorio } \\
\text { controlado }\end{array}$ & $\begin{array}{l}\mathrm{N}=121 \\
\text { Gi: } \mathrm{n}=57 \\
\mathrm{H} / \mathrm{M}=23 / 34 \\
\mathrm{Edad}=74 \\
\mathrm{Gc:}=64 \\
\mathrm{H} / \mathrm{M}=20 / 44 \\
\mathrm{Edad}=77\end{array}$ & $\begin{array}{l}\text { Ancianos } \\
\text { polimedicados } \\
\text { crónicos }\end{array}$ & Australia & $\begin{array}{l}\text { Periodo de reclutamiento: } \\
\text { 11/2000 al 12/2001 } \\
\text { Periodo de seguimiento: } \\
95 \text { días }\end{array}$ & $\begin{array}{l}\text { Visita domicilio para educar a los pacientes } \\
\text { sobre sus medicamentos, para responder } \\
\text { dudas, para mejorar la adherencia y para } \\
\text { detectar PRM }\end{array}$ & $\begin{array}{l}\text { Hubo una reducción de PRM, reingresos } \\
\text { hospitalarios y un mejor cumplimiento. Se } \\
\text { les recomendó a los médicos acciones } \\
\text { terapéuticas y las implementaron. }\end{array}$ \\
\hline $\begin{array}{l}\text { Nazareth et al. } \\
2001 \text { (48) }\end{array}$ & $\begin{array}{l}\text { Ensayo aleatorio } \\
\text { controlado }\end{array}$ & $\begin{array}{l}\text { Intervención } \\
\text { farmacéutico } \\
\text { hospitalario } \\
N=145 \\
H / M=\text { No consta } \\
\text { Edad= No consta } \\
\text { Intervención } \\
\text { farmacéutico } \\
\text { comunitario } \\
N=129 \\
H / M=\text { No consta } \\
\text { Edad= No consta }\end{array}$ & $\begin{array}{l}\text { Ancianos } \\
\text { hospitalizados }\end{array}$ & Reino Unido & $\begin{array}{l}\text { Periodo de reclutamiento: } \\
\text { 05/1995 al 03/1997 } \\
\text { Periodo de seguimiento: } \\
6 \text { meses }\end{array}$ & $\begin{array}{l}\text { Primero a los pacientes los llevó un } \\
\text { farmacéutico hospitalario y después } \\
\text { del alta un farmacéutico comunitario } \\
\text { a domicilio para ver el cambio de } \\
\text { medicación, su comprensión y la } \\
\text { adherencia. }\end{array}$ & $\begin{array}{l}\text { No hubo evidencias de mejora con este } \\
\text { plan de actuación, independientemente del } \\
\text { farmacéutico hospitalario o comunitario. }\end{array}$ \\
\hline $\begin{array}{l}\text { Raynor et al. } \\
2000(49)\end{array}$ & Estudio piloto & $\begin{array}{l}\mathrm{N}=143 \\
\mathrm{H} / \mathrm{M}=30 / 113 \\
\mathrm{Edad}=77,0 \pm 6,89\end{array}$ & $\begin{array}{l}\text { Ancianos } \\
\text { polimedicados }\end{array}$ & Reino Unido & No consta & $\begin{array}{l}\text { Se hizo una revisión a domicilio de } \\
\text { la medicación, de la adherencia, } \\
\text { identificación de PRM y se elaboró un plan } \\
\text { de acción con un seguimiento. }\end{array}$ & $\begin{array}{l}\text { Hubo una disminución en el número de } \\
\text { medicamentos recetados. Redujeron } \\
\text { los PRM y mejoró la educación de los } \\
\text { pacientes. }\end{array}$ \\
\hline $\begin{array}{l}\text { Read et al. } \\
1998(50)\end{array}$ & $\begin{array}{l}\text { Estudio descriptivo } \\
\text { transversal }\end{array}$ & $\begin{array}{l}\mathrm{N}=96 \\
\mathrm{H} / \mathrm{M}=28 / 68 \\
\mathrm{Edad}=60,4\end{array}$ & Dolor crónico & Reino Unido & $\begin{array}{l}\text { Periodo de seguimiento: } \\
\text { 4-6 semanas }\end{array}$ & $\begin{array}{l}\text { Entrevistas para calificar el dolor de los } \\
\text { pacientes y las intervenciones para aliviarlo } \\
\text { o evitar RAM e interacciones con otros } \\
\text { medicamentos. }\end{array}$ & $\begin{array}{l}\text { Se identificó AINES inapropiados, } \\
\text { medicamentos no recetados o } \\
\text { naturales con posibles interacciones y } \\
\text { duplicaciones de dosis. Se educó sobre } \\
\text { el buen uso, interacciones y dosis de sus } \\
\text { medicamentos y se les ayudó a encontrar } \\
\text { la medicación ideal para aliviar el dolor. }\end{array}$ \\
\hline $\begin{array}{l}\text { Stewart et al. } \\
1998(51)\end{array}$ & $\begin{array}{l}\text { Ensayo aleatorio } \\
\text { controlado }\end{array}$ & $\begin{array}{l}N=762 \\
\text { Gi: } n=381 \\
H / M=193 / 188 \\
\text { Edad }=66,0 \pm 15,7 \\
\text { Gc: } n=381 \\
H / M=191 / 190 \\
\text { Edad }=65,3 \pm 15,8\end{array}$ & $\begin{array}{l}\text { Ancianos } \\
\text { polimedicados } \\
\text { crónicos }\end{array}$ & EE. UU. & $\begin{array}{l}\text { Periodo de reclutamiento: } 12 \\
\text { meses } \\
\text { Periodo de seguimiento: } \\
6 \text { meses }\end{array}$ & $\begin{array}{l}\text { Visita a domicilio para asegurarse de un } \\
\text { buen manejo del medicamento, detección } \\
\text { de PRM ocultos y para aumentar la } \\
\text { vigilancia al paciente. }\end{array}$ & $\begin{array}{l}\text { Hubo una reducción de muertes fuera } \\
\text { del hospital y aunque también disminuyó } \\
\text { el porcentaje de reingresos en } 6 \text { meses, } \\
\text { después hubo un reingreso similar entre } \\
\text { los expuestos y los no expuestos a la visita } \\
\text { a domicilio. }\end{array}$ \\
\hline $\begin{array}{l}\text { Hsia Der et al. } \\
1997 \text { (52) }\end{array}$ & $\begin{array}{l}\text { Estudio prospectivo } \\
\text { con comparación } \\
\text { pre-post }\end{array}$ & $\begin{array}{l}\mathrm{N}=20 \\
\mathrm{H} / \mathrm{M}=20 / 0 \\
\mathrm{Edad}=75,1\end{array}$ & $\begin{array}{l}\text { Ancianos } \\
\text { polimedicados }\end{array}$ & EE. UU. & $\begin{array}{l}\text { Periodo de recolección de } \\
\text { datos: } \\
11 / 1994 \text { al 04/1995 } \\
\text { Periodo de reclutamiento: } 18 \\
\text { meses } \\
\text { Periodo de seguimiento: } \\
4 \text { semanas }\end{array}$ & $\begin{array}{l}\text { Revisión de medicamentos a domicilio, } \\
\text { detección de PRM, revisión de botiquines } \\
\text { y educación de su medicación en visitas } \\
\text { domiciliarias. }\end{array}$ & $\begin{array}{l}\text { Mejor cumplimiento, eliminación } \\
\text { de medicamentos caducados o } \\
\text { innecesarios, mejora de la adherencia. Los } \\
\text { farmacéuticos contribuyen a disminuir la } \\
\text { mala gestión farmacéutica en el hogar }\end{array}$ \\
\hline $\begin{array}{l}\text { Begley et al. } \\
1997 \text { (53) }\end{array}$ & Estudio piloto & $\begin{array}{l}N=190 \\
\text { Gi: } n=61 \\
H / M=24 / 37 \\
E d a d=84 \\
G c V: n=63 \\
H / M=22 / 41 \\
E d a d=81 \\
G C N V: n=66 \\
H / M=29 / 37 \\
E d a d=82\end{array}$ & $\begin{array}{l}\text { Ancianos } \\
\text { polimedicados }\end{array}$ & Reino Unido & $\begin{array}{l}\text { Periodo de reclutamiento: } \\
5 \text { meses } \\
\text { Periodo de seguimiento: } \\
12 \text { meses }\end{array}$ & $\begin{array}{l}\text { Al grupo de intervención se le revisó los } \\
\text { medicamentos a domicilio, se evaluó los } \\
\text { conocimientos del paciente, la destreza } \\
\text { para abrir las cajas de medicamentos y la } \\
\text { adherencia. Se les educó para un correcto } \\
\text { uso. }\end{array}$ & $\begin{array}{l}\text { Hubo un efecto positivo sobre el } \\
\text { conocimiento de los medicamentos y su } \\
\text { uso seguro, pero aún se podía mejorar. } \\
\text { No hubou una adherencia absoluta, pero } \\
\text { mejoró la que ya tenían los pacientes. }\end{array}$ \\
\hline \multicolumn{8}{|c|}{$\begin{array}{l}\text { PRM }=\text { Problemas relacionados con el medicamento } \\
\text { HTA = Hipertensión Arterial } \\
\text { RAM = Reacción adversa a la medicación } \\
\text { INR = Monitoreo del índice de normalización internacional } \\
\text { SNC = Sistema nervioso central } \\
\text { AINES = Fármacos antinflamatorios no esteroideos }\end{array}$} \\
\hline
\end{tabular}

Tabla 2: Análisis de la calidad metodológica de los estudios a través de los 22 puntos de valoración de la guía STROBE (8)

\begin{tabular}{|c|c|c|c|c|c|c|c|c|c|c|c|c|c|c|c|c|c|c|c|c|c|c|c|c|}
\hline \multirow[t]{2}{*}{ Artículo } & \multicolumn{24}{|c|}{ Puntuación de los puntos del cuestionario a } \\
\hline & 1 & 2 & 3 & 4 & 5 & 6 & 7 & 8 & 9 & 10 & 11 & 12 & 13 & 14 & 15 & 16 & 17 & 18 & 19 & 20 & 21 & 22 & TOTAL & $\% b$ \\
\hline Daliri et al. (10) & 1 & 1 & 1 & 1 & 1 & 0,5 & 1 & 0 & 1 & 1 & NA & 0,4 & 1 & 0,6 & 1 & 0,6 & 1 & 1 & 1 & 1 & 1 & 1 & 18,2 & 86,8 \\
\hline Papastergiou et al. (11) & 0,5 & 1 & 1 & 1 & 0 & 0,5 & 1 & 0 & 0 & 0 & NA & NA & 0 & 0,6 & 1 & 0,3 & 0 & 1 & 0 & 1 & 1 & 1 & 11 & 55 \\
\hline Fujita et al. (12) & 1 & 1 & 1 & 1 & 1 & 0,5 & 1 & 0 & 1 & 1 & NA & NA & 0,3 & 0,3 & 1 & 0 & 1 & 1 & 0 & 1 & 1 & 1 & 15,2 & 76 \\
\hline Toivo et al. (13) & 1 & 1 & 1 & 1 & 1 & 1 & 1 & 0 & 1 & 0 & NA & NA & 1 & 1 & 1 & 0,6 & 1 & 1 & 1 & 1 & 1 & 1 & 17,6 & 88 \\
\hline Kayyali et al. (14) & 0,5 & 1 & 1 & 1 & 0 & 0,5 & 1 & 1 & 0 & 0 & NA & NA & 0 & 0,6 & 1 & 0 & 1 & 1 & 0 & 0 & 1 & 1 & 11,6 & 58 \\
\hline Corsi et al. (15) & 1 & 1 & 1 & 1 & 0 & 0,5 & 1 & 0 & 0 & 0 & NA & NA & 0,3 & 0,6 & 1 & 0 & 0 & 1 & 1 & 1 & 1 & 1 & 12,5 & 62,5 \\
\hline Clifton et al. (16) & 0,5 & 1 & 1 & 1 & 1 & 0,5 & 0 & 0 & 1 & 0 & NA & NA & 0,6 & 0,6 & 1 & 0,3 & 0 & 1 & 1 & 1 & 1 & 1 & 13,6 & 68 \\
\hline Wallus et al. (17) & 1 & 1 & 1 & 1 & 0 & 0,5 & 1 & 0 & 0 & 0 & NA & NA & 1 & 0,6 & 1 & 0,6 & 1 & 1 & 1 & 1 & 1 & 1 & 14,8 & 74,1 \\
\hline Elliott et al. (18) & 1 & 1 & 1 & 1 & 0 & 0 & 0 & 0 & 1 & 0 & NA & NA & 0,3 & 0,6 & 1 & 0,6 & 1 & 1 & 1 & 1 & 1 & 1 & 13,6 & 68 \\
\hline Ong et al. (19) & 1 & 1 & 1 & 1 & 1 & 0,5 & 1 & 1 & 1 & 1 & NA & NA & 0 & 0,6 & 1 & 0,6 & 0 & 1 & 1 & 1 & 1 & 0 & 15,8 & 79 \\
\hline Basheti et al. (20) & 1 & 1 & 1 & 1 & 1 & 1 & 1 & 1 & 1 & 1 & NA & NA & 1 & 0,6 & 1 & 0 & 1 & 1 & 1 & 1 & 1 & 1 & 18,6 & 93 \\
\hline
\end{tabular}




\begin{tabular}{|c|c|c|c|c|c|c|c|c|c|c|c|c|c|c|c|c|c|c|c|c|c|c|c|c|}
\hline \multirow[t]{2}{*}{ Artículo } & \multicolumn{24}{|c|}{ Puntuación de los puntos del cuestionario a } \\
\hline & 1 & 2 & 3 & 4 & 5 & 6 & 7 & 8 & 9 & 10 & 11 & 12 & 13 & 14 & 15 & 16 & 17 & 18 & 19 & 20 & 21 & 22 & TOTAL & $\% \mathbf{b}$ \\
\hline Steele et al. (21) & 1 & 1 & 1 & 1 & 0 & 1 & 1 & 0 & 1 & 0 & NA & NA & 0,6 & 0,6 & 1 & 0 & 0 & 1 & 1 & 1 & 1 & 0 & 13,3 & 66,5 \\
\hline Kalista et al. (22) & 0,5 & 1 & 1 & 1 & 1 & 0,5 & 1 & 0 & 1 & 0 & NA & NA & 0,3 & 0,6 & 1 & 0 & 0 & 1 & 1 & 1 & 1 & 1 & 14 & 70 \\
\hline Carter et al. (23) & 0,5 & 1 & 1 & 1 & 1 & 0,5 & 1 & 0 & 1 & 1 & NA & NA & 0 & 0,6 & 1 & 0,6 & 1 & 1 & 1 & 1 & 1 & 1 & 16,3 & 81,5 \\
\hline Pherson et al. (24) & 1 & 1 & 1 & 1 & 0 & 0,5 & 1 & 0 & 0 & 0 & NA & NA & 0,6 & 0,6 & 1 & 0 & 1 & 1 & 1 & 0 & 1 & 0 & 11,8 & 59 \\
\hline Reidt et al. (25) & 0 & 1 & 1 & 1 & 1 & 1 & 1 & 1 & 0 & 0 & NA & NA & 0 & 0,6 & 1 & 0,6 & 1 & 1 & 1 & 1 & 0 & 1 & 14,3 & 71,5 \\
\hline Kogut et al. (26) & 1 & 1 & 1 & 1 & 0 & 0,5 & 1 & 0 & 1 & 0 & NA & NA & 0,6 & 0,6 & 1 & 0,3 & 0 & 1 & 1 & 0 & 1 & 1 & 13,2 & 66 \\
\hline Kalyango et al. (27) & 1 & 1 & 1 & 1 & 0 & 0,5 & 1 & 0 & 1 & 0 & NA & NA & 0 & 0,6 & 1 & 0,6 & 0 & 1 & 0 & 1 & 1 & 1 & 12,8 & 64 \\
\hline El Hajj et al. (28) & 0,5 & 1 & 1 & 1 & 0 & 0,5 & 1 & 0 & 1 & 0 & NA & NA & 0,3 & 0,6 & 1 & 0 & 1 & 1 & 1 & 1 & 1 & 1 & 14 & 70 \\
\hline Kwint et al. (29) & 1 & 1 & 1 & 1 & 1 & 0,5 & 1 & 0 & 1 & 0 & NA & NA & 1 & 0,6 & 1 & 0,6 & 1 & 1 & 1 & 1 & 1 & 1 & 16,8 & 84 \\
\hline Lee et al. (30) & 1 & 1 & 1 & 1 & 1 & 0,5 & 1 & 0 & 0 & 0 & NA & NA & 0,6 & 0,6 & 1 & 0 & 1 & 1 & 1 & 1 & 1 & 1 & 14,8 & 74 \\
\hline Elliott et al. (31) & 1 & 1 & 1 & 0 & 1 & 1 & 1 & 0 & 0 & 1 & NA & NA & 1 & 0,6 & 1 & 0,6 & 0 & 1 & 1 & 1 & 1 & 1 & 15,3 & 76,6 \\
\hline Stafford et al. (33) & 1 & 1 & 1 & 1 & 1 & 0,5 & 1 & 0 & 1 & 0 & NA & NA & 0 & 0,6 & 1 & 0,3 & 0 & 1 & 1 & 0 & 1 & 1 & 13,5 & 67,5 \\
\hline Fikri-Benbrahim et al. (32) & 1 & 1 & 1 & 1 & 1 & 1 & 1 & 1 & 1 & 1 & 1 & 0,8 & 0,6 & 1 & 1 & 0,3 & 1 & 1 & 1 & 1 & 1 & 1 & 20,8 & 94,5 \\
\hline Sheridan et al. (34) & 1 & 1 & 1 & 1 & 1 & 0 & 1 & 0 & 1 & 0 & NA & NA & 0 & 0,6 & 1 & 0 & 1 & 1 & 1 & 1 & 1 & 1 & 14,6 & 73 \\
\hline Roughead et al. (35) & 1 & 1 & 1 & 1 & 1 & 1 & 1 & 0 & 0 & 0 & NA & NA & 0 & 0,6 & 1 & 0,3 & 0 & 1 & 1 & 1 & 1 & 1 & 14 & 70 \\
\hline Flanagan et al. (36) & 0,5 & 1 & 1 & 0 & 1 & 0,5 & 1 & 1 & 0 & 0 & NA & NA & 0,6 & 0,6 & 1 & 0,3 & 0 & 1 & 1 & 1 & 1 & 0 & 12,6 & 63 \\
\hline Hugtenburg et al. (37) & 1 & 1 & 1 & 1 & 1 & 0,5 & 1 & 1 & 0 & 1 & NA & NA & 0 & 0,6 & 1 & 0 & 1 & 1 & 1 & 1 & 1 & 1 & 16,1 & 80,5 \\
\hline Moultry et al. (38) & 1 & 1 & 1 & 1 & 0 & 0,5 & 1 & 0 & 1 & 0 & NA & NA & 0,3 & 0,6 & 1 & 0 & 0 & 1 & 1 & 1 & 1 & 1 & 13,5 & 67,5 \\
\hline Holland et al. (39) & 1 & 1 & 1 & 0 & 0 & 1 & 1 & 1 & 0 & 1 & NA & NA & 1 & 0,6 & 1 & 0,6 & 1 & 1 & 1 & 0 & 1 & 1 & 15,3 & 76,5 \\
\hline Salter et al. (40) & 1 & 1 & 1 & 1 & 0 & 0,5 & 1 & 0 & 1 & 1 & NA & NA & 0 & 0,6 & 1 & 0 & 0 & 1 & 1 & 1 & 1 & 1 & 14,2 & 71 \\
\hline Triller et al. (41) & 0,5 & 1 & 1 & 1 & 0 & 1 & 1 & 1 & 0 & 1 & NA & NA & 0,3 & 0,6 & 1 & 0,3 & 0 & 1 & 1 & 1 & 1 & 1 & 14,8 & 74 \\
\hline Sorensen et al. (42) & 1 & 1 & 1 & 1 & 0 & 0,5 & 1 & 0 & 0 & 1 & NA & NA & 0 & 0,6 & 1 & 0,6 & 0 & 1 & 1 & 0 & 1 & 0 & 11,8 & 59 \\
\hline Zillich et al. (43) & 0,5 & 1 & 1 & 1 & 0 & 1 & 1 & 1 & 1 & 0 & NA & NA & 0 & 0,6 & 1 & 0,3 & 0 & 1 & 1 & 1 & 1 & 1 & 14,5 & 72,5 \\
\hline Sorensen et al. (44) & 1 & 1 & 1 & 1 & 1 & 1 & 1 & 0 & 1 & 1 & NA & NA & 1 & 0,6 & 1 & 0,6 & 1 & 1 & 1 & 1 & 1 & 1 & 18,3 & 91,5 \\
\hline Peterson et al. (45) & 1 & 1 & 1 & 1 & 1 & 0,5 & 1 & 1 & 1 & 1 & NA & NA & 1 & 0,6 & 1 & 0,6 & 0 & 1 & 1 & 1 & 1 & 1 & 17,8 & 89 \\
\hline Triller et al. (46) & 0,5 & 1 & 1 & 1 & 0 & 0,5 & 1 & 0 & 1 & 0 & NA & NA & 0 & 0,3 & 1 & 0 & 1 & 1 & 1 & 0 & 1 & 1 & 12,3 & 61,5 \\
\hline Naunton et al. (47) & 1 & 1 & 1 & 0 & 1 & 1 & 1 & 0 & 0 & 0 & NA & NA & 0,6 & 0,6 & 1 & 0,3 & 1 & 1 & 1 & 1 & 1 & 1 & 15,6 & 78 \\
\hline Nazareth et al. (48) & 1 & 1 & 1 & 1 & 1 & 1 & 1 & 1 & 1 & 0 & NA & NA & 1 & 0,6 & 1 & 0,6 & 0 & 1 & 0 & 1 & 1 & 1 & 16,3 & 81,5 \\
\hline Raynor et al. (49) & 0,5 & 1 & 1 & 1 & 0 & 1 & 1 & 0 & 0 & 0 & NA & NA & 0,6 & 0,6 & 1 & 0 & 0 & 1 & 1 & 0 & 1 & 1 & 11,8 & 59 \\
\hline Read et al. (50) & 0,5 & 1 & 1 & 1 & 0 & 0,5 & 1 & 0 & 1 & 0 & NA & NA & 0,6 & 0,6 & 1 & 0,3 & 1 & 1 & 0 & 0 & 1 & 1 & 12,6 & 63 \\
\hline Stewart et al. (51) & 1 & 1 & 1 & 1 & 1 & 1 & 1 & 0 & 1 & 0 & NA & NA & 0 & 0,6 & 1 & 0,6 & 1 & 1 & 1 & 1 & 1 & 1 & 16,3 & 81,5 \\
\hline Hsia Der et al. (52) & 1 & 1 & 0 & 1 & 1 & 0,5 & 1 & 0 & 1 & 0 & NA & NA & 0 & 0,6 & 1 & 0,6 & 1 & 1 & 1 & 1 & 1 & 0 & 13,8 & 69 \\
\hline Begley et al. (53) & 0,5 & 1 & 0 & 1 & 1 & 1 & 1 & 1 & 1 & 1 & NA & NA & 0,6 & 0,6 & 1 & 0,3 & 0 & 0 & 1 & 1 & 1 & 0 & 14,1 & 70,5 \\
\hline
\end{tabular}




\section{DISCUSIÓN}

La revisión realizada muestra la existencia de investigaciones que pondrían en valor el rol del farmacéutico en la atención de la salud a domicilio. La revisión de la medicación y de los botiquines son las actividades más frecuentemente descritas, y permiten detectar, prevenir y/o resolver Problemas Relacionados con los Medicamentos (PRM), carencias de información, adiestrar al paciente en el manejo de los medicamentos y dispositivos rutinarios de medida y promocionar la adherencia.

El análisis de la actualidad/obsolescencia de los estudios incluidos demostró la plena vigencia del tema estudiado ya que los datos calculados mostraron menor obsolescencia que los datos usuales en el ámbito de los estudios sobre hospitalización domiciliaria (62).

La evaluación de la calidad de los estudios incluidos en este trabajo mediante STROBE no resultó muy elevada, aunque análoga a la observada en otras revisiones similares $(63,64)$.

El diseño de los estudios revisados mostró una adecuada evidencia las recomendaciones de la U. S. Agency for Healthcare Research and Quality (AHRQ). El optar por el diseño observacional se debió a la búsqueda de una consistente relación causa-efecto, ya que se buscaban trabajos de intervención (9). En consecuencia, se encontró un predominio de los trabajos con diseño descriptivo transversal, aunque también se revisó un buen número de ensayos clínicos.

Era predecible la filiación anglófona de una mayoría de artículos y que estuvieran redactados, principalmente, en lengua inglesa, este idioma es el elegido para la publicación de la mayoría de los artículos ya que hacerlo en otra lengua distinta resulta negativo para la visibilidad. Asimismo, el número de revistas anglófonas contenidas en las principales bases de datos bibliográficas es muy elevado y publicar en ellas facilita la citación $(63,65)$.

La población incluida en los estudios presentó edades avanzadas. Esta situación es común en las unidades de $\mathrm{HaD}$ ya que para grupos de población adulta o anciana con patologías crónicas o terminales la $\mathrm{HaD}$ encuentra todo su significado y ha demostrado ser un modelo eficiente. El desarrollo de esquemas de hospitalización domiciliaria integral es una oportunidad como alternativa a la hospitalización convencional adaptada a pacientes ancianos en nuestro entorno asistencial $(63,66)$. En cuanto al período de seguimiento resultó adecuado para valorar los resultados de la intervención, requisito que cumplieron la mayoría de los estudios seleccionados. Se considera necesario un periodo de varias semanas para poder valorar los resultados (64).

La mayoría de los estudios se centraron en personas polimedicadas, hecho que está en consonancia con el objetivo del estudio y con las características de la HaD, donde la principal población ingresada es adulta o anciana con patologías crónicas $(66,67)$.

El perfil de estos pacientes, ancianos y polimedicados, hace que presenten un mayor riesgo de morbi-mortalidad, lo que justificaría la búsqueda proactiva de oportunidades de mejora por parte del farmacéutico en las visitas domiciliarias, a través de servicios diferenciados como los que se han descrito en este trabajo. Una reciente revisión sistemática (68) evidenciaba una elevada prevalencia de PRM entre los pacientes ancianos que viven en sus casas y sugiere la necesidad de intervenciones efectivas que mejoren el uso de los medicamentos en esta población. Por su parte, Talebreza \& McPherson (69), en su trabajo "reconocimiento y gestión de la polifarmacia en enfermedades avanzadas", concluyeron que los adultos mayores, la mayoría de los pacientes incluidos en los estudios revisados, corrían un mayor riesgo de PRM al soportar una gran carga de polifarmacia $y$, en consecuencia, los profesionales que atienden a esta población vulnerable debían de utilizar habilidades de comunicación para intervenir en estas personas y sus familias con el fin de mejorar su medicación y evitar los PRM. Anderson et al. (70), en otra reciente revisión sistemática, observaron que esta intervención farmacéutica tenía un impacto medible en beneficio de las personas a las que se controlaba su medicación. 
A la vista de los resultados, la revisión de la medicación podría ser por tanto una actividad eficiente para mejorar la epidemiología de PRM en este perfil de pacientes. Estos resultados muestran que la revisión de la medicación efectuada desde la farmacia comunitaria motivó un mejor conocimiento del plan farmacoterapéutico y de la medicación, lo cual favorece la adherencia y es requisito ineludible para alcanzar los objetivos de la farmacoterapia. Este servicio profesional aborda, de manera global, los problemas de salud y los medicamentos que utiliza el paciente, centrándose en la valoración de la necesidad, efectividad y seguridad de la farmacoterapia (71).

Requiere, además, la aplicación de medidas y estrategias que permitan corregir las situaciones detectadascomo: estrategias técnicas, educativas, conductuales, tratamiento de observación directa, técnicas de apoyo social, técnicas dirigidas a profesionales y recuerdo de las estrategias (72).

Una de las intervenciones más frecuentes, en esta revisión, han sido aquellas relacionadas con la adherencia al tratamiento farmacológico. La falta de adherencia es un problema relacionado con el proceso de uso de los medicamentos que puede dar lugar, si no se soluciona, a resultados negativos asociados a la medicación, como la pérdida de la efectividad y otros problemas no deseados. Como se ha probado en esta revisión, la intervención del farmacéutico permite detectar y/o mejorar estas situaciones.

Las estrategias para mejorar la adherencia al tratamiento farmacológico van a procurar que haya coincidencia entre las instrucciones dadas por el profesional sanitario respecto a la medicación y la realización de éstas por parte del paciente (72). La visita domiciliaria podría ser un momento idóneo para diseñar intervenciones efectivas en adherencia, por estar el paciente en su entorno natural y por la posibilidad de conocer sus hábitos y rutinas y adaptarlos a las necesidades relacionadas con la medicación. En este sentido, la experiencia del paciente con su medicación y la adaptación individualizada a su forma de vida han demostrado tener un impacto positivo sobre la adherencia y son aspectos a tener en cuenta en el plan de cuidados del paciente (73). Como también se ha podido comprobar, se apreció un mejor conocimiento y uso de los medicamentos tras la intervención farmacéutica. Es fundamental que las intervenciones incluyan elementos educativos, que impliquen no solo una adecuada comunicación, sino también una atención especial a la motivación, que debe ser más intensa si se pretende mantener en el tiempo el nuevo hábito. Una vez consolidada esta actitud, habrá que hacer hincapié en medidas de soporte que refuercen la base cognoscitiva y conceptual ya lograda (74).

Un importante beneficio a la hora de la educación de los pacientes, según Catania (75), es que el farmacéutico comunitario es el profesional de la salud más confiable. Debido a esta confianza los pacientes respondían, en su mayoría, positivamente a los consejos y la formación brindada por los farmacéuticos. Estos profesionales pueden colaborar con otros sanitarios de atención domiciliaria para coordinar la atención y garantizar la continuidad farmacoterapéutica.

Otra de las intervenciones observadas coincidió con Pellegrin et al. (76), donde confirmó que estas acciones reducían la tasa de hospitalización relacionada con la medicación para adultos mayores y producían un ahorro en la medicación. En el entorno domiciliario, la educación del paciente y del cuidador en el manejo de la medicación y la intervención de la farmacia comunitaria en el monitoreo de pacientes puede ser clave para mejorar la adherencia farmacoterapéutica (4).

Posibles limitaciones de esta revisión:

Una importante limitación de la presente revisión fue no poder recuperar el texto completo de algunos artículos, siendo los principales motivos no estar digitalizados en la web de la revista, no aparecer en las principales colecciones de revistas e incluso no poder ser recuperado a través de la red de bibliotecas universitarias.

La evaluación de la calidad documental de los estudios y los grados de evidencia y recomendación quizá recomendaran la eliminación de algún artículo recogido en esta revisión. Además, es 
cierto que algunos diseños no reflejaron adecuadamente la intervención realizada desde la farmacia comunitaria, o bien podían considerarse artículos de opinión o protocolos de futuros estudios. De todos modos, no se eliminó ningún trabajo para no limitar información a los posibles lectores de esta revisión y, en consecuencia, disponer de todas las posibles intervenciones que se pueden realizar desde la farmacia comunitaria.

Con respecto a los resultados de las intervenciones observadas, hubiera sido interesante resumirlas en un valor cuantitativo o haber dado una medida de tendencia central. Sin embargo, no fue posible al no encontrar datos cuantitativos en la mayoría de los artículos revisados y a la gran heterogeneidad los mismos.

Por todo lo anteriormente expuesto, se pudo concluir que la intervención farmacéutica en pacientes de HaD, principalmente la revisión de la medicación y botiquines aporta beneficios en la población anciana polimedicada, al permitir la detección, prevención y/o resolución de los PRM, promoción de la adherencia farmacoterapéutica, y la mejora en el almacenamiento y conservación de los medicamentos en el domicilio del paciente. No obstante, serían necesarios más ensayos clínicos que pongan de manifiesto el verdadero valor añadido de las intervenciones farmacéuticas, y su comparación con otras intervenciones en salud.

\section{BIBLIOGRAFÍA}

1. Cool L, Missiaen J, Vandijck D, Lefebvre T, Lycke M, De Jonghe PJ, et al. An observational pilot study to evaluate the feasibility and quality of oncological home-hospitalization. Eur $\mathrm{J}$ Oncol Nurs. 2019;40:44-52. DOI: 10.1016/j.ejon.2019.03.003; PMID: 31229206

2. Raphaël R, Yves D, Giselle C, Magali M, Odile CM. Cancer treatment at home or in the hospital: what are the costs for French public health insurance? Findings of a comprehensive-cancer centre. Health Policy. 2005;72(2):141-8. DOI: 10.1016/j.healthpol.2004.07.001; PMID: 15802149

3. Diaz Gegundez M, Gomez de Argila I, Ferrer Cobo E, Castelar Delgado E. 10 años de hospitalización a domicilio en el entorno de un hospital comarcal. Hosp Domic. 2020;4(2):19-30. DOI: 10.22585/hospdomic.v4i2.96

4. Gea Cabrera A, Sanz-Lorente M, Sanz-Valero J, López-Pintor E. Compliance and Adherence to Enteral Nutrition Treatment in Adults: A Systematic Review. Nutrients. 2019;11(11):pii E2627. DOI: 10.3390/nu11112627; PMID: 31684024

5. Costa D, Van C, Abbott $P$, Krass I. Investigating general practitioner engagement with pharmacists in Home Medicines Review. J Interprof Care. 2015;29(5):469-75. DOI: 10.3109/13561820.2015.1012253; PMID: 25692718

6. Kucukarslan SN, Hagan AM, Shimp LA, Gaither CA, Lewis NJW. Integrating medication therapy management in the primary care medical home: A review of randomized controlled trials. Am J Health Syst Pharm. 2011;68(4):335-45. DOI: 10.2146/ajhp100405; PMID: 21289329

7. Haynes RB, McDonald H, Garg AX, Montague P. Interventions for helping patients to follow prescriptions for medications. Cochrane Database Syst Rev. 2002;(2):CD000011. DOI: 10.1002/14651858.CD000011; PMID: 12076376

8. von Elm E, Altman DG, Egger M, Pocock SJ, Gøtzsche PC, Vandenbroucke JP. Declaración de la Iniciativa STROBE (Strengthening the Reporting of Observational studies in Epidemiology): directrices para la comunicación de estudios observacionales. Gac Sanit. 2008;22(2):144-50. DOI: 10.1157/13119325; PMID: 18420014 
9. Berkman ND, Lohr KN, Ansari M, McDonagh M, Balk E, Whitlock E, et al. Grading the Strength of a Body of Evidence When Assessing Health Care Interventions for the Effective Health Care Program of the Agency for Healthcare Research and Quality: An Update. In: Methods Guide for Effectiveness and Comparative Effectiveness Reviews [monograph on the Internet]. Rockville (MD), USA: Agency for Healthcare Research and Quality; 2008 [cited April 3, 2020]. (AHRQ Methods for Effective Health Care). Disponible en: http://www.ncbi.nlm.nih.gov/books/ NBK174881/

10. Daliri S, Hugtenburg JG, Ter Riet G, van den Bemt BJF, Buurman BM, Scholte Op Reimer WJM, et al. The effect of a pharmacy-led transitional care program on medication-related problems post-discharge: A before-After prospective study. PLOS ONE. 2019;14(3):e0213593. DOI: 10.1371/journal.pone.0213593; PMID: 30861042

11. Papastergiou J, Luen M, Tencaliuc S, Li W, van den Bemt B, Houle S. Medication management issues identified during home medication reviews for ambulatory community pharmacy patients. Can Pharm J (Ott). 2019;152(5):334-42. DOI: 10.1177/1715163519861420; PMID: 31534588

12. Fujita K, Kushida K, Moles RJ, Chen TF. Home healthcare professionals' perspectives on quality dimensions for home pharmaceutical care in Japan. Geriatr Gerontol Int. 2019;19(1):35-43. DOI: 10.1111/ggi.13562; PMID: 30556238

13. Toivo T, Airaksinen M, Dimitrow M, Savela E, Pelkonen K, Kiuru V, et al. Enhanced coordination of care to reduce medication risks in older home care clients in primary care: a randomized controlled trial. BMC Geriatr. 2019;19(1):332. DOI: 10.1186/s12877-019-1353-2; PMID: 31775650

14. Kayyali R, Funnell G, Harrap N, Patel A. Can community pharmacy successfully bridge the gap in care for housebound patients? Res Social Adm Pharm. 2019;15(4):425-39. DOI: 10.1016/j. sapharm.2018.06.011; PMID: 30917894

15. Corsi K, Lemay V, Orr KK, Cohen L. Pharmacist medication therapy management in home health care: Investigation of a sustainable practice model. J Am Pharm Assoc. 2018;58(4S):S64-8. DOI: 10.1016/j.japh.2018.04.028; PMID: 29887258

16. Clifton CL, Branham AR, Hayes HH, Moose JS, Rhodes LA, Marciniak MW. Financial impact of patients enrolled in a medication adherence program at an independent community pharmacy. J Am Pharm Assoc. 2018;58(4S):S109-13. DOI: 10.1016/j.japh.2018.04.022; PMID: 30006182

17. Walus AN, Woloschuk DMM. Impact of Pharmacists in a Community-Based Home Care Service: A Pilot Program. Can J Hosp Pharm. 2017;70(6):435-42. DOI: 10.4212/cjhp.v70i6.1718; PMID: 29299003

18. Elliott RA, Lee CY, Beanland C, Goeman DP, Petrie N, Petrie B, et al. Development of a clinical pharmacy model within an Australian home nursing service using co-creation and participatory action research: the Visiting Pharmacist (ViP) study. BMJ Open. 2017;7(11):e018722. DOI: 10.1136/bmjopen-2017-018722; PMID: 29102998

19. Ong KY, Cheen MHH, Chng JSG, Chen LL, Ng SM, Lim SH, et al. Effectiveness of a multidisciplinary home-based medication review program in reducing healthcare utilization among older adult Singaporeans: Home-based medication reviews for the elderly. Geriatr Gerontol Int. 2017;17(2):302-7. DOI: 10.1111/ggi.12706; PMID: 26790376

20. Basheti IA, Al-Qudah RA, Obeidat NM, Bulatova NR. Home medication management review in outpatients with chronic diseases in Jordan: a randomized control trial. Int J Clin Pharm. 2016;38(2):404-13. DOI: 10.1007/s11096-016-0266-9; PMID: 26960406 
21. Steele KM, Ruisinger JF, Bates J, Prohaska ES, Melton BL, Hipp S. Home-Based Comprehensive Medication Reviews: Pharmacist's Impact on Drug Therapy Problems in Geriatric Patients. Consult Pharm. 2016;31(10):598-605. DOI: 10.4140/TCP.n.2016.598; PMID: 27725070

22. Kalista T, Lemay V, Cohen L. Postdischarge community pharmacist-provided home services for patients after hospitalization for heart failure. J Am Pharm Assoc. 2015;55(4):438-42. DOI: 10.1331/JAPhA.2015.14235; PMID: 26161487

23. Carter SR, Moles R, White L, Chen TF. The impact of patients' perceptions of the listening skills of the pharmacist on their willingness to re-use Home Medicines Reviews: A structural equation model. Res Social Adm Pharm. 2015;11(2):163-75. DOI: 10.1016/j.sapharm.2014.07.002; PMID: 25681299

24. Pherson EC, Shermock KM, Efird LE, Gilmore VT, Nesbit T, LeBlanc Y, et al. Development and implementation of a postdischarge home-based medication management service. Am J Health Syst Pharm. 2014;71(18):1576-83. DOI: 10.2146/ajhp130764; PMID: 25174018

25. Reidt SL, Larson TA, Hadsall RS, Uden DL, Blade MA, Branstad R. Integrating a pharmacist into a home healthcare agency care model: impact on hospitalizations and emergency visits. Home Healthc Nurse. 2014;32(3):146-52. DOI: 10.1097/NHH.0000000000000024; PMID: 24584311

26. Kogut SJ, Goldstein E, Charbonneau C, Jackson A, Patry G. Improving medication management after a hospitalization with pharmacist home visits and electronic personal health records: an observational study. Drug Healthc Patient Saf. 2014;6:1-6. DOI: 10.2147/DHPS.S56574; PMID: 24465136

27. Kalyango JN, Hall M, Karamagi C. Home medication management practices and associated factors among patients with selected chronic diseases in a community pharmacy in Uganda. BMC Health Serv Res. 2012;12:323. DOI: 10.1186/1472-6963-12-323; PMID: 22988920

28. El Hajj MS, El-Ajez RH, Al-Ismail MSM, Sawaftah DM. Home diagnostic tests in the state of Qatar: a snapshot of public views, level of awareness and use. Int J Clin Pharm. 2012;34(6):885-92. DOI: 10.1007/s11096-012-9687-2; PMID: 22890990

29. Kwint HF, Faber A, Gussekloo J, Bouvy ML. The contribution of patient interviews to the identification of drug-related problems in home medication review. J Clin Pharm Ther. 2012;37(6):67480. DOI: 10.1111/j.1365-2710.2012.01370.x; PMID: 22861493

30. Lee CY, George J, Elliott RA, Stewart K. Exploring stakeholder perspectives on medication review services for older residents in retirement villages. Int J Pharm Pract. 2012;20(4):249-58. DOI: 10.1111/j.2042-7174.2012.00189.x; PMID: 22775521

31. Elliott RA, Martinac G, Campbell S, Thorn J, Woodward MC. Pharmacist-led medication review to identify medication-related problems in older people referred to an Aged Care Assessment Team: a randomized comparative study. Drugs Aging. 2012;29(7):593-605. DOI: 10.1007/ bf03262276; PMID: 22715865

32. Fikri-Benbrahim N, Faus MJ, Martínez-Martínez F, Alsina DG-S, Sabater-Hernández D. Effect of a pharmacist intervention in Spanish community pharmacies on blood pressure control in hypertensive patients. Am J Health Syst Pharm. 2012;69(15):1311-8. DOI: 10.2146/ajhp110616; PMID: 22821790

33. Stafford L, van Tienen EC, Bereznicki LRE, Peterson GM. The benefits of pharmacist-delivered warfarin education in the home. Int J Pharm Pract. 2012;20(6):384-9. DOI: 10.1111/j.20427174.2012.00217.x; PMID: 23134097

34. Sheridan J, Butler R, Brandt T, Harrison J, Jensen M, Shaw J. Patients' and pharmacists' perceptions of a pilot Medicines Use Review service in Auckland, New Zealand: Medicines Use 
Review pilot in New Zealand. J Pharm Health Serv Res. 2012;3(1):35-40. DOI: 10.1111/j.17598893.2011.00075.x

35. Roughead EE, Barratt JD, Ramsay E, Pratt N, Ryan P, Peck R, et al. Collaborative home medicines review delays time to next hospitalization for warfarin associated bleeding in Australian war veterans. J Clin Pharm Ther. 2011;36(1):27-32. DOI: 10.1111/j.1365-2710.2009.01149.x; PMID: 21108651

36. Flanagan $\mathrm{P}$, Virani $\mathrm{A}$, Baker $\mathrm{W}$, Roelants $\mathrm{H}$. Pharmacists making house calls: innovative role or overkill? Can J Hosp Pharm. 2010;63(6):412-9. DOI: 10.4212/cjhp.v63i6.959; PMID: 22479013

37. Hugtenburg JG, Borgsteede SD, Beckeringh JJ. Medication review and patient counselling at discharge from the hospital by community pharmacists. Pharm World Sci. 2009;31(6):630-7. DOI: 10.1007/s11096-009-9314-Z; PMID: 19649720

38. Moultry AM, Poon IO. Perceived value of a home-based medication therapy management program for the elderly. Consult Pharm. 2008;23(11):877-85. DOI: 10.4140/tcp.n.2008.877; PMID: 19072012

39. Holland R, Brooksby I, Lenaghan E, Ashton K, Hay L, Smith R, et al. Effectiveness of visits from community pharmacists for patients with heart failure: HeartMed randomised controlled trial. BMJ. 2007;334(7603):1098. DOI: 10.1136/bmj.39164.568183.AE; PMID: 17452390

40. Salter C, Holland R, Harvey I, Henwood K. «I haven't even phoned my doctor yet.» The advice giving role of the pharmacist during consultations for medication review with patients aged 80 or more: qualitative discourse analysis. BMJ. 2007;334(7603):1101. DOI: 10.1136/ bmj.39171.577106.55; PMID: 17449504

41. Triller DM, Hamilton RA. Effect of pharmaceutical care services on outcomes for home care patients with heart failure. Am J Health Syst Pharm. 2007;64(21):2244-9. DOI: 10.2146/ajhp050492; PMID: 17959576

42. Sorensen L, Stokes JA, Purdie DM, Woodward M, Roberts MS. Medication management at home: medication risk factor prevalence and inter-relationships. J Clin Pharm Ther. 2006;31(5):485-91. DOI: 10.1111/j.1365-2710.2006.00768.x; PMID: 16958827

43. Zillich AJ, Sutherland JM, Kumbera PA, Carter BL. Hypertension outcomes through blood pressure monitoring and evaluation by pharmacists (HOME study). J Gen Intern Med. 2005;20(12):1091-6. DOI: 10.1111/j.1525-1497.2005.0226.x; PMID: 16423096

44. Sorensen L, Stokes JA, Purdie DM, Woodward M, Elliott R, Roberts MS. Medication reviews in the community: results of a randomized, controlled effectiveness trial. $\mathrm{Br} \mathrm{J}$ Clin Pharmacol. 2004;58(6):648-64. DOI: 10.1111/j.1365-2125.2004.02220.x; PMID: 15563363

45. Peterson GM, Fitzmaurice KD, Naunton M, Vial JH, Stewart K, Krum H. Impact of pharmacistconducted home visits on the outcomes of lipid-lowering drug therapy. J Clin Pharm Ther. 2004;29(1):23-30. DOI: 10.1046/j.1365-2710.2003.00532.x; PMID: 14748894

46. Triller DM, Clause SL, Briceland LL, Hamilton RA. Resolution of drug-related problems in home care patients through a pharmacy referral service. Am J Health Syst Pharm. 2003;60(9):905-10. DOI: 10.1093/ajhp/60.9.905; PMID: 12756941

47. Naunton M, Peterson GM. Evaluation of Home-Based Follow-Up of High-Risk Elderly Patients Discharged from Hospital. J Pharm Pract Res. 2003;33(3):176-82. DOI: 10.1002/jppr2003333176

48. Nazareth I, Burton A, Shulman S, Smith P, Haines A, Timberal H. A pharmacy discharge plan for hospitalized elderly patients--a randomized controlled trial. Age Ageing. 2001;30(1):33-40. DOI: 10.1093/ageing/30.1.33; PMID: 11322670 
49. Raynor DK, Nicolson M, Nunney J, Petty D, Vail A, Davies L. The development and evaluation of an extended adherence support programme by community pharmacists for elderly patients at home. Int J Pharm Pract. 2000;8(3):157-64. DOI: 10.1111/j.2042-7174.2000.tb01001.x

50. Read RW, Krska J. Targeted medication review: patients in the community with chronic pain. Int J Pharm Pract. 1998;6(4):216-22. DOI: 10.1111/j.2042-7174.1998.tb00940.x

51. Stewart S, Pearson S, Luke CG, Horowitz JD. Effects of home-based intervention on unplanned readmissions and out-of-hospital deaths. J Am Geriatr Soc. 1998;46(2):174-80. DOI: 10.1111/ j.1532-5415.1998.tb02535.x; PMID: 9475445

52. Hsia Der E, Rubenstein LZ, Choy GS. The benefits of in-home pharmacy evaluation for older persons. J Am Geriatr Soc. 1997;45(2):211-4. DOI: 10.1111/j.1532-5415.1997.tb04510.x; PMID: 9033522

53. Begley S, Livingstone C, Hodges N, Williamson V. Impact of domiciliary pharmacy visits on medication management in an elderly population. Int J Pharm Pract. 1997;5(3):111-21. DOI: 10.1111/j.2042-7174.1997.tb00895.x

54. Toivo T, Dimitrow M, Puustinen J, Savela E, Pelkonen K, Kiuru V, et al. Coordinating resources for prospective medication risk management of older home care clients in primary care: procedure development and RCT study design for demonstrating its effectiveness. BMC Geriatr. 2018;18(1):74. DOI: 10.1186/s12877-018-0737-z; 10.1186/s12877-018-0737-z

55. Stafford L, Peterson GM, Bereznicki LRE, Jackson SL, van Tienen EC, Angley MT, et al. Clinical outcomes of a collaborative, home-based postdischarge warfarin management service. Ann Pharmacother. 2011;45(3):325-34. DOI: 10.1345/aph.1P617; PMID: 21386021

56. Papastergiou J, Zervas J, Li W, Rajan A. Home medication reviews by community pharmacists: Reaching out to homebound patients. Can Pharm J (Ott). 2013;146(3):139-42. DOI: 10.1177/1715163513487830; PMID: 23795196

57. Lenaghan E, Holland R, Brooks A. Home-based medication review in a high risk elderly population in primary care--the POLYMED randomised controlled trial. Age Ageing. 2007;36(3):292-7. DOI: 10.1093/ageing/afm036; PMID: 17387123

58. Holland R, Lenaghan E, Harvey I, Smith R, Shepstone L, Lipp A, et al. Does home based medication review keep older people out of hospital? The HOMER randomised controlled trial. BMJ. 2005;330(7486):293. DOI: 10.1136/bmj.38338.674583.AE; PMID: 15665005

59. Holland R, Lenaghan E, Smith R, Lipp A, Christou M, Evans D, et al. Delivering a home-based medication review, process measures from the HOMER randomised controlled trial. Int $\mathrm{J}$ Pharm Pract. 2006;14(1):71-9. DOI: 10.1211/ijpp.14.1.0009

60. Roughead EE, Barratt JD, Ramsay E, Pratt N, Ryan P, Peck R, et al. The effectiveness of collaborative medicine reviews in delaying time to next hospitalization for patients with heart failure in the practice setting: results of a cohort study. Circ Heart Fail. 2009;2(5):424-8. DOI: 10.1161/ CIRCHEARTFAILURE.109.861013; PMID: 19808372

61. Stewart S, Pearson S, Horowitz JD. Effects of a home-based intervention among patients with congestive heart failure discharged from acute hospital care. Arch Intern Med. 1998;158(10):106772. DOI: 10.1001/archinte.158.10.1067; PMID: 9605777

62. Sanz-Valero J, Wanden-Berghe C. Análisis bibliométrico de la producción científica, indizada en MEDLINE, sobre los servicios de salud proporcionados por las unidades de hospitalización a domicilio. Hosp Domic. 2017;1(1):21-34. DOI: 10.22585/hospdomic.v1i1.3 
63. Álvarez Velásquez S, Sanz Valero J. Ventajas de la quimioterapia domiciliaria en los enfermos adultos con neoplasias: revisión sistemática. Hosp Domic. 2020;4(1):25-41. DOI: 10.22585/hospdomic.v4i1.98

64. Aracil-Lavado E, Wanden-Berghe C, Sanz-Valero J. Evaluación de la calidad de vida según el estado nutricional del paciente paliativo adulto: revisión sistemática. Hosp Domic. 2017;1(4):199210. DOI: 10.22585/hospdomic.v1i4.27

65. Campos Cañuelo D, Sanz-Valero J, Wanden-Berghe C. Consecuencias de la nutrición parenteral domiciliaria en adultos con síndrome de intestino corto: revisión exploratoria. Hosp Domic. 2019;3(2):149-62. DOI: 10.22585/hospdomic.v3i2.60

66. Mas MÀ, Santaeugènia S. Hospitalización domiciliaria en el paciente anciano: revisión de la evidencia y oportunidades de la geriatría. Revista Española de Geriatría y Gerontología. 2015;50(1):26-34. DOI: 10.1016/j.regg.2014.04.003

67. Estrada Cuxart O, Massa Domínguez B, Ponce González MÁ, Mirón Rubio M, Torres Corts A, Mujal Martínez A, et al. Proyecto HAD 2020: una propuesta para consolidar la hospitalización a domicilio en España. Hosp Domic. 2017;1(2):93-117. DOI: 10.22585/hospdomic.v1i2.13

68. Plácido Al, Herdeiro MT, Morgado M, Figueiras A, Roque F. Drug-related Problems in Homedwelling Older Adults: A Systematic Review. Clin Ther 2020 Apr;42(4):559-572.e14. DOI: 10.1016/j.clinthera.2020.02.005; PMID: 32147147

69. Talebreza S, McPherson ML. Recognizing and Managing Polypharmacy in Advanced Illness. Med Clin North Am. 2020;104(3):405-13. DOI: 10.1016/j.mcna.2019.12.003; PMID: 32312406

70. Anderson LJ, Schnipper JL, Nuckols TK, Shane R, Le MM, Robbins K, et al. Effect of medication reconciliation interventions on outcomes: A systematic overview of systematic reviews. Am J Health Syst Pharm. 2019;76(24):2028-40. DOI: 10.1093/ajhp/zxz236; PMID: 31789354

71. Sabater Hernández D, Silva Castro MM, Faus Dáder MJ. Método Dáder: Guía de Seguimiento Farmacoterapéutico. 3a edición. Granada, España: Grupo de Investigación en Atención Farmacéutica, Universidad de Granada; 2007.

72. Rodríguez Chamorro MA, Pérez Merino EM, García Jiménez E, Rodríguez Chamorro A, Martínez Martínez F, Faus Dader MJ. Revisión de estrategias utilizadas para la mejora de la adherencia al tratamiento farmacológico. Pharm Care Esp. 2014;16(3):110-20.

73. Mohammed MA, Moles RJ, Chen TF. Medication-related burden and patients' lived experience with medicine: a systematic review and metasynthesis of qualitative studies. BMJ Open 2016; 6(2):e010035. DOI: 10.1136/bmjopen-2015-010035; DOI: 10.1136/bmjopen-2015-010035

74. Rodríguez Chamorro MÁ, García-Jiménez E, Amariles P, Rodríguez Chamorro A, Pérez Merino EM, Martínez Martínez F, et al. Efecto de la actuación farmacéutica en la adherencia del tratamiento farmacológico de pacientes ambulatorios con riesgo cardiovascular (Estudio EMDADERCV-INCUMPLIMIENTO). Aten Primaria. 2011;43(5):245-53. DOI: 10.1016/j.aprim.2010.05.006; PMID: 21211865

75. Catania PN. What is the role of the pharmacist in home care? Home Care Provid. 1997;2(5):22931. DOI: 10.1016/s1084-628x(97)90115-5; PMID: 9362678

76. Pellegrin KL, Krenk L, Oakes SJ, Ciarleglio A, Lynn J, McInnis T, et al. Reductions in Medication-Related Hospitalizations in Older Adults with Medication Management by Hospital and Community Pharmacists: A Quasi-Experimental Study. J Am Geriatr Soc. 2017;65(1):212-9. DOI: 10.1111/jgs.14518; PMID: 27714762 